\title{
Manipulating Deformable Linear Objects: Attachable Adjustment-Motions for Vibration Reduction
}

\author{
Shigang YUE ${ }^{1}$ and Dominik HENRICH \\ Embedded Systems and Robotics (RESY) \\ Informatics Faculty, University of Kaiserslautern \\ 67653 Kaiserslautern, Germany \\ Http://resy.informatik.uni-kl.de/ \\ [shigang, henrich]@informatik.uni-kl.de
}

\begin{abstract}
This paper addresses the problem of handling deformable linear objects (DLOs) in a suitable way to avoid acute vibration. Different types of adjustment-motions that eliminate vibration of deformable objects and can be attached to the end of an arbitrary end-effector's trajectory are presented. For describing the dynamics of deformable linear objects, the finite element method is used to derive the dynamic differential equations. Genetic algorithm is used to find the optimal adjustment motion for each simulation example. Experiments are conducted to verify the presented manipulating method.
\end{abstract}

Keywords: deformable object, manipulation, motion planning, robot, vibration

\section{Introduction}

The automated handling and assembly of materials have been studied by many researchers in the areas of manufacturing, robotics, and artificial intelligence. Until now, most studies assume that the objects to be manipulated are rigid. However, deformable materials such as cables, wires, ropes, cloths, rubber tubes, sheet metals, paper sheets and leather products can be found almost everywhere in the real world of industry and human life. In most cases, deformable materials and parts are still handled and assembled by humans. Practical methods for the automatic handling and manipulation of deformable objects are urgently required.

Previous research work involving the modeling and controlling of DLOs such as beams, cables, wires, and tubes etc. has been found, for example in ${ }^{[1-13]}$. There are two basic methods for handling DLOs; one is the force-based method with physical model ${ }^{[1,4,5,8,10,12]}$, the other is the vision-based modeless method ${ }^{[2,7]}$. Some researchers are trying to use hybrid methods (i.e. force and vision sensors or other sensors) to cope with the linear deformable materials ${ }^{[3,6]}$. On the other hand, Zheng et al. ${ }^{[13]}$ derived strategies to insert a flexible beam into a hole without sensors, while Hirai et al. ${ }^{[9,11]}$ presented human skillful transplantation method. However, the above methods are specialized and confined to limited applications.

When a robot executes a manipulation task, its motion can be divided into several motion primitives, each of which has a particular target state to be achieved in the task context. These primitives are called 'skills'. An adequately defined skill can have generality to be applied to various similar tasks. Until now, most of the research work on skill-based manipulation deals with rigid objects ${ }^{[14,15]}$.

\footnotetext{
${ }^{1}$ S.G. YUE is a research fellow of the Alexander von Humboldt Foundation from Beijing Polytechnic University
} 
Skill-based manipulation for handling deformable linear materials has been touched upon recently. For example, Henrich et. al ${ }^{[16]}$ analyzed the contact states and point contacts of DLOs with regard to manipulation skills, Abegg et. al ${ }^{[17]}$ studied the contact state transitions based on force and vision sensors and Remde et.al ${ }^{[18]}$ discussed the problem of picking-up DLOs by experimentation.

However, the effects of dynamic vibration are not taken into account in the skill-related work described above. The dynamic effects of deformable objects can not be neglected, especially when the objects are moved quickly by a robot arm. As shown in Figure 1, the uncertainty resulting from oscillation may cause failure in the insert-into-hole operation. Therefore, the vibration caused by inertia should be depressed during the motion or eliminated as soon as possible after the motion.

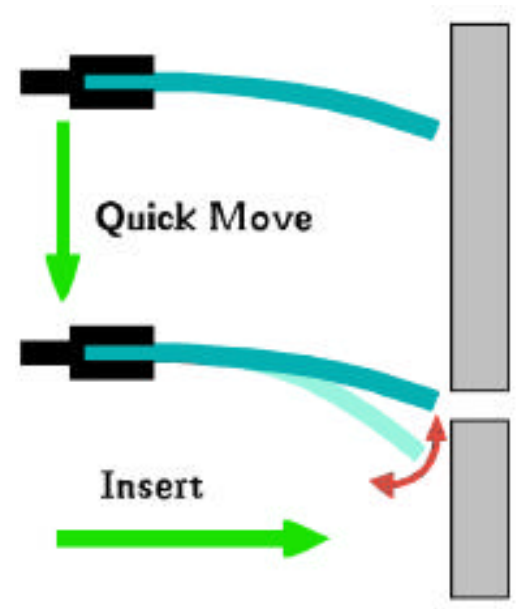

Figure 1. The quick operation causes uncertainty and failure

Vibration reduction of flexible structures has been a research topic for many researchers, and the previous works have been reviewed by Chen et al. ${ }^{[19]}$. Chen et al. also present a passive approach based on open-loop concept for vibration-free handling of deformable beams; similar ideas can be found in ${ }^{[20,21]}$, which deal only with rigid bodies. However, application of the method presented by ${ }^{[19]}$ is limited due to its stable start condition and a relatively simple trajectory. Considering the complex manipulations involved in practical situations, such as avoiding obstacles, picking-up and insert-into-hole etc., stable start condition cannot be satisfied easily.

In this paper, we present attachable adjustment-motion at the end of an arbitrary trajectory of the end-effector. This attachable adjustment-motion can be treated as one of the vibration-free manipulation skills. Vibration caused during the arbitrary previous trajectory can be reduced during the attached adjustment-motion. Our approach also uses an open-loop concept. The suitable adjustment motion can be found by applying optimization methods. Additionally, the adjustment-motion is chosen to be as simple as possible, so that it may be easily utilized.

The rest of this paper consists of five parts. First, a finite element model for describing the dynamics of deformable linear objects is presented, wherein gravity is also taken into account. Second, the adjustment-motion is specified. Third, a method that uses genetic algorithms for generating the adjustment-motion is given. Then, several cases based on the introduced model 
and method are presented. Finally, an experiment is conducted to verify the presented manipulating method.

\section{Dynamic Modeling of DLOs}

The dynamic behavior of DLOs varies and is dependent upon the type of material involved; for example, cables are different from rubber bars. Furthermore, the behavior also depends on the length of the DLOs ${ }^{[16]}$. Finite element methods will be used in this paper to describe the dynamics of deformable linear objects. Only the elastic deformation of DLOs is considered, plastic deformation will be disregarded.

Figure 2 shows a generalized deformable element used here with eight parameters. The transverse deflections of the element are modeled by a quintic polynomial and the longitudinal deflections assumed to be a linear polynomial ${ }^{[22,23]}$. The coordinates of the element are assembled in a vector form as

$$
\overrightarrow{\phi_{e}}=\left(\begin{array}{llll}
\phi_{1} & \phi_{2} & \cdots & \phi_{8}
\end{array}\right)^{T}
$$

where $\phi_{1}$ and $\phi_{5}$ are the axial displacements along the $x$-axis, $\phi_{2}$ and $\phi_{6}$ are the transverse displacements along the $y$-axis, $\phi_{3}$ and $\phi_{7}$ are the rotary displacements about the $z$-axis, and $\phi_{4}$ and $\phi_{8}$ are the curvature displacements in the $x y$ plane.

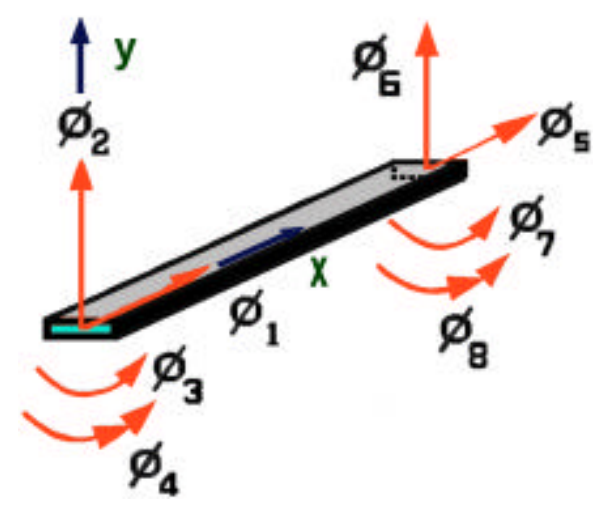

Figure 2. Parameters of a generalized element

The longitudinal deformation $D_{x e}$ and the transverse deformation $D_{y e}$ at an arbitrary point $p$ on the axis of an element can be expressed as follows,

$$
\begin{aligned}
& D_{x e}=\vec{\phi}_{e}^{T} \vec{D}_{x} \\
& D_{y e}=\vec{\phi}_{e}^{T} \vec{D}_{y}
\end{aligned}
$$


where $\vec{D}_{x}$ and $\vec{D}_{y}$ are the vectors of the interpolation polynomials and can be found in the appendix.

Before the dynamic equation can be obtained, it is necessary to derive the kinetic and potential energy of the element.

The velocity at point $p$ is given by

$$
\vec{V}_{p}=\left[\begin{array}{ll}
\vec{i} & \vec{j}
\end{array}\right]\left[\begin{array}{c}
V_{a x}-\dot{q} D_{y e}+\dot{D}_{x e} \\
V_{a y}+\dot{q}\left(x+D_{x e}\right)+\dot{D}_{y e}
\end{array}\right]
$$

where $\vec{i}$, and $\vec{j}$ are the unit vectors of the floating coordinate system with $x$ and $y$ as axis and point $a$ as origin, and $V_{a x}$ and $V_{a y}$ are the projections of velocity at point $a$ on the $\mathrm{x}$ and $\mathrm{y}$ axis, respectively.

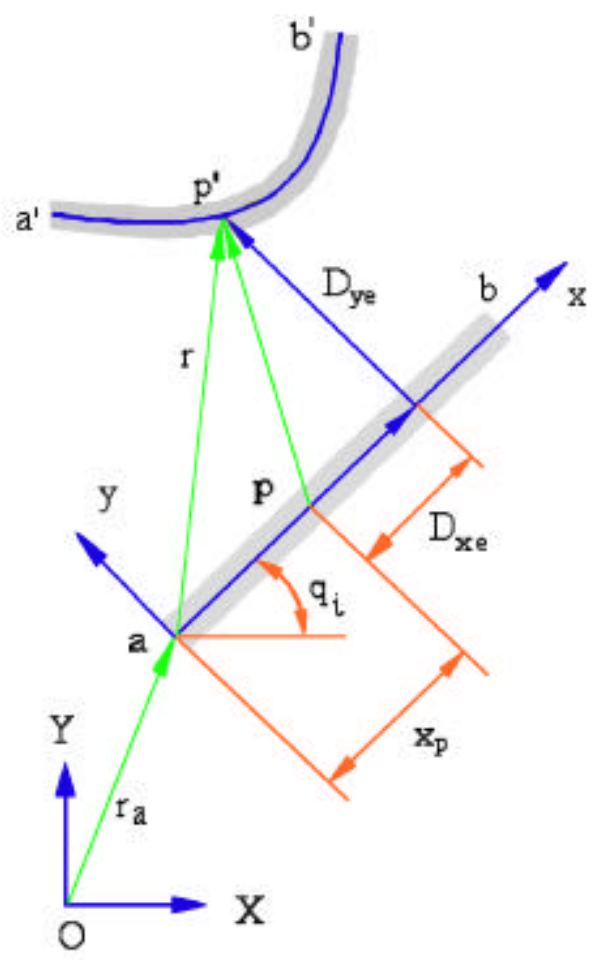

Figure 3. The deformation of a DLO element

The kinetic energy of a DLO element can be expressed as

$$
T=\frac{1}{2} \int_{0}^{L} \lambda \vec{V}_{p}^{2} d x
$$

where $\lambda$ is the mass of unit length, and $L$ is the length of the element. Substitution of equations (2) and (3) into equation (4), and then substitution of equation (4) into equation (5) with subsequent rearrangement yields: 


$$
\begin{gathered}
T=\frac{1}{2} \lambda\left(V_{a x}^{2} L+V_{a y}^{2} L+\frac{1}{3} \dot{q}^{2} L^{3}+V_{a y} \dot{q} L^{2}\right)+\frac{1}{2} \lambda \dot{q}^{2} \frac{I L}{A} \\
+\frac{1}{2} \dot{q}^{2} \vec{\phi}_{e}^{T}[m] \overrightarrow{\phi_{e}}+\frac{1}{2} \dot{\vec{\phi}}_{e}^{T}\left[m^{+}\right] \dot{\overrightarrow{\phi_{e}}}+\dot{q} \dot{\vec{\phi}}_{e}^{T}[B] \overrightarrow{\phi_{e}} \\
+\vec{\phi}_{e}^{T}\left\{y^{+}\right\}+\dot{\vec{\phi}}_{e}^{T}\left\{x^{+}\right\}+\dot{\dot{q}} \dot{\vec{\phi}}_{e}^{T}\left\{z^{+}\right\}
\end{gathered}
$$

where

$$
\begin{gathered}
{\left[m^{+}\right]=[m]+\left[m_{z}\right]} \\
{[m]=\lambda \int_{0}^{L}\left(\vec{D}_{y} \vec{D}_{y}^{T}+\vec{D}_{x} \vec{D}_{x}^{T}\right) d x} \\
{[B]=2 \lambda \int_{0}^{L}\left(\vec{D}_{y} \vec{D}_{x}^{T}\right) d x} \\
{\left[m_{z}\right]=\frac{\lambda}{A} \int_{0}^{L}\left(\frac{\partial \vec{D}_{y}}{\partial x}\right)\left(\frac{\partial \vec{D}_{y}^{T}}{\partial x}\right) d x} \\
\left\{y^{+}\right\}=\lambda \int_{0}^{L}\left(\dot{q}^{2} x \vec{D}_{x}+V_{a y} \dot{q} \vec{D}_{x}-V_{a x} \dot{q} \vec{D}_{y}\right) d x \\
\left\{x^{+}\right\}=\lambda \int_{0}^{L}\left(\dot{q} y \vec{D}_{y}+V_{a y} \vec{D}_{y}+V_{a x} \vec{D}_{x}\right) d x \\
{\left[z^{+}\right]=\frac{\lambda}{A} \int_{0}^{L}\left(\frac{\partial \vec{D}_{y}}{\partial x}\right) d x}
\end{gathered}
$$

The potential energy of an element is the sum of the strain energy and the energy due to gravity,

$$
\begin{aligned}
E_{p} & =\frac{1}{2} \int_{0}^{L}\left\{E I\left(\frac{\partial^{2} D_{y e}}{\partial x^{2}}\right)^{2}+\frac{(E I)^{2}}{G A^{*}}\left(\frac{\partial^{3} D_{y e}}{\partial x^{3}}\right)^{2}+E A\left(\frac{\partial D_{x e}}{\partial x}\right)^{2}\right\} d x \\
& +\lambda g\left\{r_{a y}+\frac{1}{2} L \sin q_{i}+\int_{0}^{L}\left(D_{y e} \cos q_{i}+D_{x e} \sin q_{i}\right) d x\right\}
\end{aligned}
$$

where $r_{a y}$ is the geometric projection of $r_{a}$ (see Figure 3), $r_{a}$ is a vector extending from the global frame to the beam fixed frame, $E$ and $G$ represent elastic and shear modulus respectively, $A^{*}$ is the shear cross-sectional area, and $g$ is the gravitational acceleration vector. With substitution of equations (2) and (3) into equation (14), then

$$
\begin{gathered}
E_{p}=\frac{1}{2} \vec{\phi}_{e}^{T}[K] \vec{\phi}_{e} \\
+\lambda g\left\{r_{a y}+\frac{1}{2} L \sin q_{i}+\int_{0}^{L} \vec{\phi}_{e}^{T}\left(\vec{D}_{y} \cos q_{i}+\vec{D}_{x} \sin q_{i}\right) d x\right\}
\end{gathered}
$$

The Lagrange equation for a DLO element is expressed as

$$
\frac{d}{d t}\left(\frac{\partial E_{k}}{\partial \dot{\overrightarrow{\phi_{e}}}}\right)-\frac{\partial E_{k}}{\partial \vec{\phi}_{e}}+\frac{\partial E_{p}}{\partial \vec{\phi}_{e}}=\vec{q}_{e}+\vec{f}
$$


Substituting equations (6) and (15) into equation (16) and rearranging into a compact form gives

$$
\left[m_{e}\right] \ddot{\overrightarrow{\phi_{e}}}+\left[c_{e}\right] \dot{\overrightarrow{\phi_{e}}}+\left[k_{e}\right] \overrightarrow{\phi_{e}}=\vec{p}_{e}+\vec{f}_{e}
$$

where

$$
\begin{gathered}
{\left[m_{e}\right]=[m]+\left[m_{z}\right]} \\
{\left[c_{e}\right]=2 \dot{q}[B]} \\
{\left[k_{e}\right]=\dot{q}[B]-\dot{q}^{2}[m]+[k]} \\
\vec{p}_{e}=\vec{y}^{+}-\vec{x}^{+}-\vec{z}^{+}-\vec{m}_{g} \\
\vec{m}_{g}=\lambda g \int_{0}^{L}\left(\vec{D}_{y} \cos q_{i}+\vec{D}_{x} \sin q_{i}\right) d x
\end{gathered}
$$

and $\vec{f}_{e}$ is the external force given by the adjacent element.

It is evident that the damping matrix here is only determined by symmetric mass matrix and the element's angular velocity. However, the damping effects are quite various for different DLOs; therefore the damping matrix of the element should be modified in order to deal with different DLOs. The following equations are introduced:

$$
\left[c_{e}\right]^{\prime}=\left[c_{e}\right]+[c]_{m}
$$

where $[c]_{m}$ is the modifying matrix which can be obtained as

$$
[c]_{m}=\xi\left[m_{e}\right]\left[\begin{array}{lll}
\ddots & & 0 \\
& \sqrt{k_{i i} / m_{i i}} & \\
0 & & \ddots
\end{array}\right]_{8 \times 8}
$$

where $\xi$ is the damping coefficient, which can be determined by estimation or experiment, $k_{i i}$ and $m_{i i}$ are the diagonal elements of $\left[k_{e}\right]$ and $\left[m_{e}\right]$, respectively. Based on equations (23) and (24), we have modified elemental dynamic equation

$$
\left[m_{e}\right] \ddot{\vec{\phi}}+\left[c_{e}\right]^{\prime} \dot{\bar{\phi}}+\left[k_{e}\right] \vec{\phi}_{e}=\vec{p}_{e}+\vec{f}_{e}
$$

The elemental dynamic equations can be assembled into a dynamic system and expressed in terms of global variables:

$$
[M]_{s}\{\ddot{\Phi}\}+[C]_{s}\{\dot{\Phi}\}+[K]_{s}\{\Phi\}=\{P\}_{s}
$$

where $[M]_{s},[C]_{s}$, and $[K]_{s}$ are respectively the mass, damping, and stiffness matrix of the system and $\{P\}_{s}$ is the load vector of the system.

The quasi-static equation which only describes the stable deformations can be obtained as

$$
[K]_{s}\{\Phi\}=\{P\}_{s}
$$




\section{Adjustment-Motion of End-effector}

In a normal motion, DLOs go through an acceleration, constant velocity, and deceleration mode. Both acceleration and deceleration will cause vibration of DLOs. To avoid the vibration, a special trajectory can be designed. Most previous research involves attempts to damp the vibration during the complete trajectory ${ }^{[19]}$.

With regard to manipulation, only the vibration which could result in the failure of the next operation should be eliminated. Most important of all, the vibration eliminating method should be applicable in similar cases without necessitating major changes to the method. Based on the above requirements, we present attachable adjustment-motions that can be conducted at the end of any arbitrary trajectory to damp the vibration caused by this previous motion. Adjustmentmotion here refers to a kind of agile motion with limited scope.

\subsection{Adjustment-Motion Classification}

The attachable adjustment-motions can be sorted into two different groups according to their motion styles. One is translation-adjustment-motion (TAMo) and another one is rotationadjustment-motion (RAMo). The adjustment-motions can also be classified either one-way adjustment-motion or two-way adjustment-motion. The different kinds of adjustment-motions presented in the paper can be found in Table 1.

\section{Table 1. Classification of Adjustment-Motions}

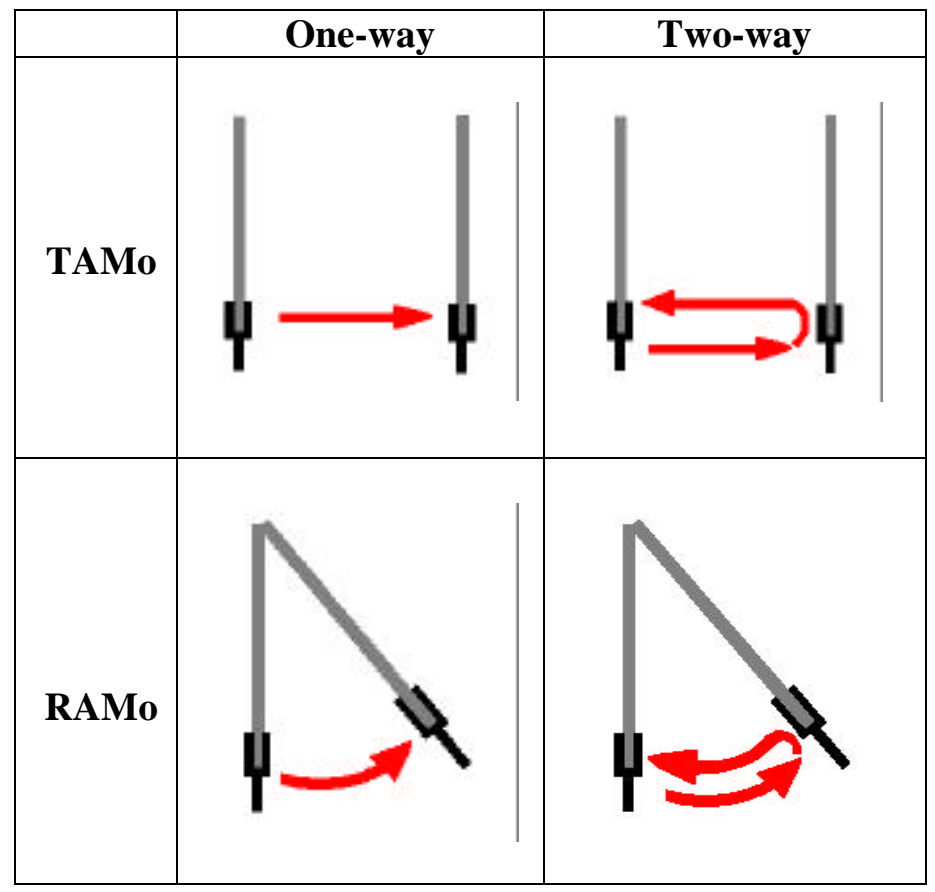

\subsection{One-way Adjustment-Motion Description}


A one-way RAMo is presented as an example to describe adjustment-motions in detail. As shown in Figure 4, the adjustment-motion moves along a circle which assumes the nominal endpoint of the DLO as its center and the length of the DLO as its radius. The adjustment-motion starts from the last nominal position of the previous arbitrary trajectory and ends at a certain point of the circle.

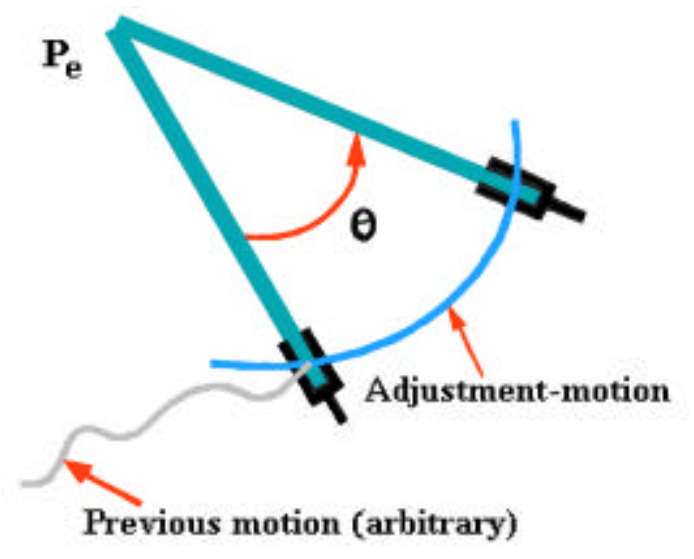

Figure 4. An one-way RAMo

The profile of the one-way RAMo can be written as

$$
\begin{aligned}
& x=L \cos \theta+x_{e d} \\
& y=L \sin \theta+y_{e d}
\end{aligned}
$$

where $x_{e d}$ and $y_{e d}$ describe the nominal position of the endpoint $p_{e}\left(x_{e d}, y_{e d}\right)$ of the DLO. This point also acts as the center of the adjustment motion, and

$$
\theta=\omega t
$$

where $\omega$ is the constant angular velocity of the DLO during the adjustment.
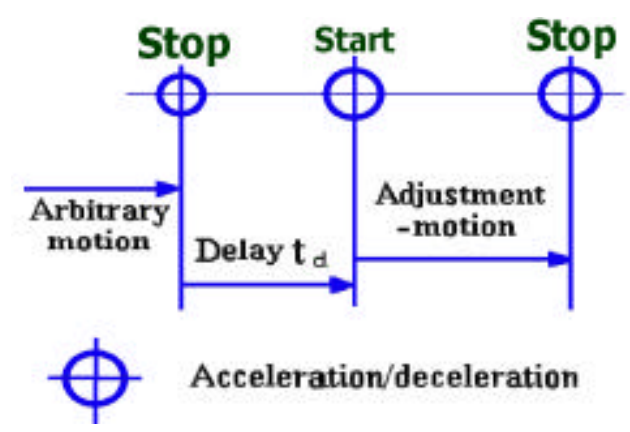

Figure 5. Scheme of an one-way RAMo 
By using this adjustment motion (Figure 5), the DLO will be accelerated from rest to a velocity $\omega$. Then it is moved at this constant velocity for a certain period $t_{a}$. Deceleration is performed after this period to stop the adjustment motion.

There is a delay time between the previous arbitrary end-effector's trajectory and adjustmentmotion, as shown in Figure 5. The delay is useful in enabling the adjustment to take advantage of inertia. The optimal delay time can be determined by optimization methods. It should be noted that the constant velocity is the angular velocity for the DLO's circular motion.

For industrial robots, the time of acceleration and deceleration $\Delta t$ is quite small. It is reasonable to assume that the movement of the DLOs involves a sharp increase of the acceleration and deceleration (Figure 6).

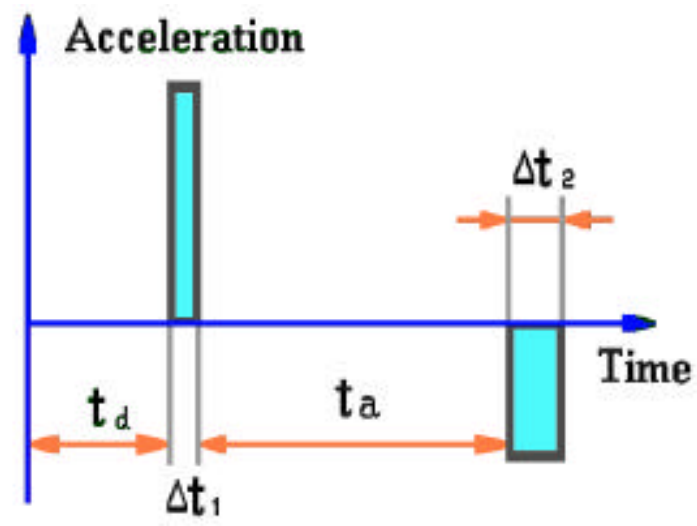

Figure 6. Acceleration profile of an one-way RAMo

The relationships between acceleration (deceleration) and constant velocity are

$$
\omega=\varepsilon_{a c c} \Delta t_{1}
$$

and

$$
\omega=-\varepsilon_{\text {dec }} \Delta t_{2}
$$

This implies that only two of the three parameters in equations (31) or (32) must be determined. The parameters of adjustment-motion which should be determined by optimization are as follows: delay time $t_{d}$, value of acceleration (negative is also possible) $\varepsilon_{a c c}$, the constant angular velocity $\omega$, the running time of this velocity $t_{a}$ and the value of deceleration $\varepsilon_{d e}$.

The one-way TAMo can be defined in a similar way.

\subsection{Two-way Adjustment-Motion}

When a robot manipulates a DLO, perhaps it is expected to return the DLO to its previous position as the final task in order to start next operation. Two-way adjustment-motion is presented to meet this demand.

If a two-way adjustment-motion (either RAMo or TAMo) is conducted, the DLO will be handled and finally returned to the original position with the same posture after adjustment. A two-way 
adjustment-motion can be defined as two connected symmetrical parts as shown in Figure 7, and each part is an one-way adjustment-motion. One may realize that a two-way adjustment-motion and an one-way adjustment-motion have the same number of parameters that should be determined by optimization methods. The details of a two-way adjustment-motion thus must not be described again.

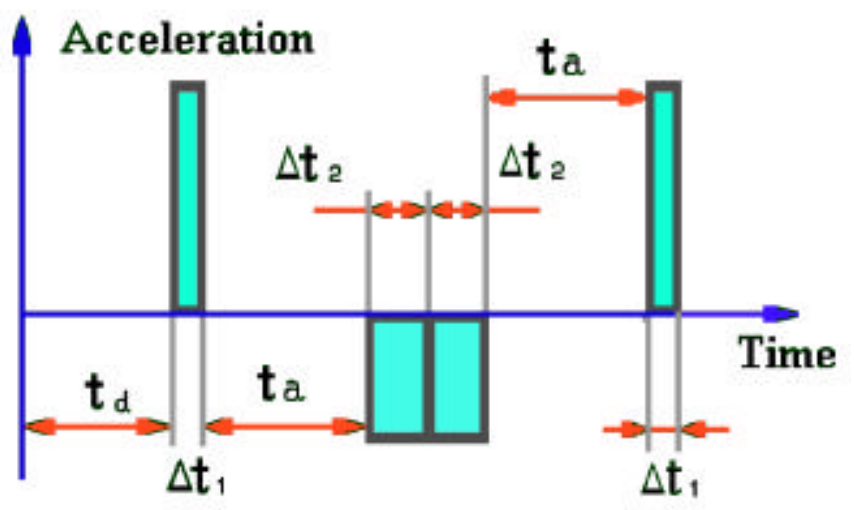

Figure 7. Acceleration profile of a two-way adjustment

\section{Adjustment-Motion Generation by Genetic Algorithms}

As described in the above chapter, the adjustment-motion should be carefully selected in order to attain the aim of vibration reduction. Genetic Algorithms (GAs), are evolutionary, stochastic and global search methods. Their performance is superior to those of classical techniques ${ }^{[24,25]}$ and they have been used successfully in robot path planning ${ }^{[26,27]}$. There has been little work reported involving application of this optimization method to trajectory generation for handling DLOs.

\subsection{Objective and Constraint Functions}

Since gravity is taken into account in this method, as stated above, the handling operation is not limited to the horizontal plane. The oscillation may not be symmetric with respect to its nominal position, therefore the parameter which describes the amplitude of vibration is redefined here, as shown in Figure 8.

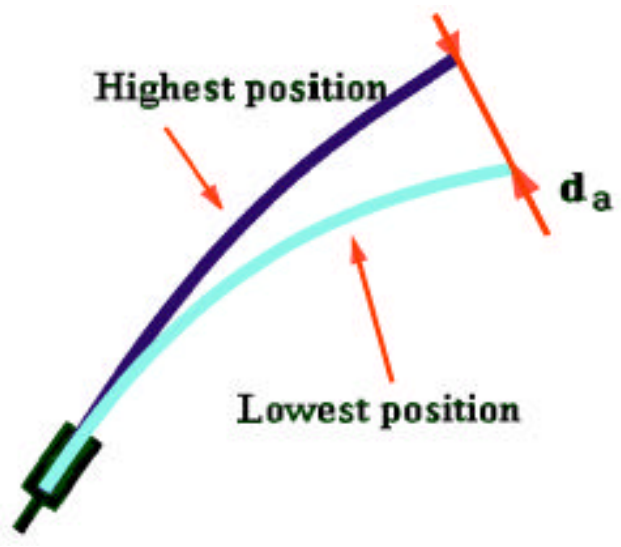

Figure 8. Vibrational amplitude of a DLO 
The following objective function is minimized according to the defined parameter:

$$
f=d_{a}
$$

where $d_{a}$ is the maximum endpoint vibrational amplitude of a DLO as described in Figure 8 .

Since the adjustment-motion can not start before the delay time $t_{d}$, it is reasonable to account for the $d_{a}$ immediately after the delay period.

The adjustment motion planning problem for the vibration-free handling of DLOs can now be expressed as the following optimization problem:

$$
\begin{aligned}
\min : f=d_{a} \\
\text { s.t. } t_{d, \min } \leq t_{d} \leq t_{d, \max } \\
\varepsilon_{a c, \min } \leq \varepsilon_{a c} \leq \varepsilon_{a c, \max } \\
\omega_{\min } \leq \omega \leq \omega_{\max } \\
t_{c, \text { min }} \leq t_{c} \leq t_{c, \max } \\
\varepsilon_{d e, \min } \leq \varepsilon_{d e} \leq \varepsilon_{d e, \max }
\end{aligned}
$$

where $t_{d, \text { min }}$ and $t_{d \text {,max }}$ are the lower and upper permitted delay times, $\varepsilon_{a c, \min }$ and $\varepsilon_{a c, \text { max }}$ are the lower and upper permitted DLO angular accelerations, $\omega_{\min }$ and $\omega_{\max }$ are the lower and upper permitted DLO constant angular velocities, $t_{c, \min }$ and $t_{c, \max }$ are the lower and upper permitted running times at the constant angular velocity along the adjustment circle, and $\varepsilon_{d e \text {,min }}$ and $\varepsilon_{d e \text {, max }}$ are the lower and upper DLO angular decelerations, respectively. The lower and upper limits of equations (35b), (35c) and (35e) are determined by the capabilities of the robot. The maximum possible scope of adjustment-motions is determined mainly by the upper limits of equation (35d).

\subsection{Optimization Method}

As stated above, since the basic form of adjustment-motion is given, if the parameters of the adjustment-motion have been determined, then the attached adjustment can be easily determined. We use genetic algorithms (GAs) to determine the adjustment-motion parameters. GA programs can be found described in detail ${ }^{[25]}$. The GA procedure proposed to optimize adjustment-motion for handling of DLOs without serious residual vibration is shown in Figure 9.

In the procedure, the coding method for the parameters is binary coding, which has been shown to be the most effective coding method for this type of parameter optimization ${ }^{[25]}$. The fitness function of the optimization is selected as being the maximum amplitude of deflection shortly after the delay period, which is the same as the objective function equation (34).

As shown in Figure 9, initialization randomly generates an initial host population $P_{0}$. New generations are formed by survivors from the last generation and new individuals generated 
through mutation and crossover. Single-point crossover is used to form the new generation. The adjustment-motion is finally decided when the termination condition is satisfied. The termination condition of the procedure can be maximum generations or certain value according to next operation, such as the diameter of a hole through which the DLO is to be inserted.

\section{Procedure AMDLO BEGIN \\ $\mathrm{N}:=0$; \\ Initialize $\left(P_{N}\right)$; \\ Evaluate $\left(P_{N}\right)$; \\ REPEAT}

Selection 2 parents from $P_{N}$;

Crossover $\left(P_{N}\right)$;

Mutation $\left(P_{N}\right)$;

Form new generation $P_{N}$;

Evaluate $\left(P_{N}\right)$;

$\mathrm{N}:=\mathrm{N}+1$;

UNTIL Termination Condition = True;

Select adjustment-motion for DLO;

\section{END}

\section{Figure 9. Procedure to generate adjustment-motion for handling DLOs}

\section{Case Studies}

In these case studies, we use a long DLO described by two elements (Figure 10). One of its ends, which is grasped by the end-effector, is treated as a cantilever without vibrational deflection to exclude curvature displacement. The other distal end is treated as a free-end. Therefore, we use a total of eight general coordinates to describe the deformed shape of the DLO.

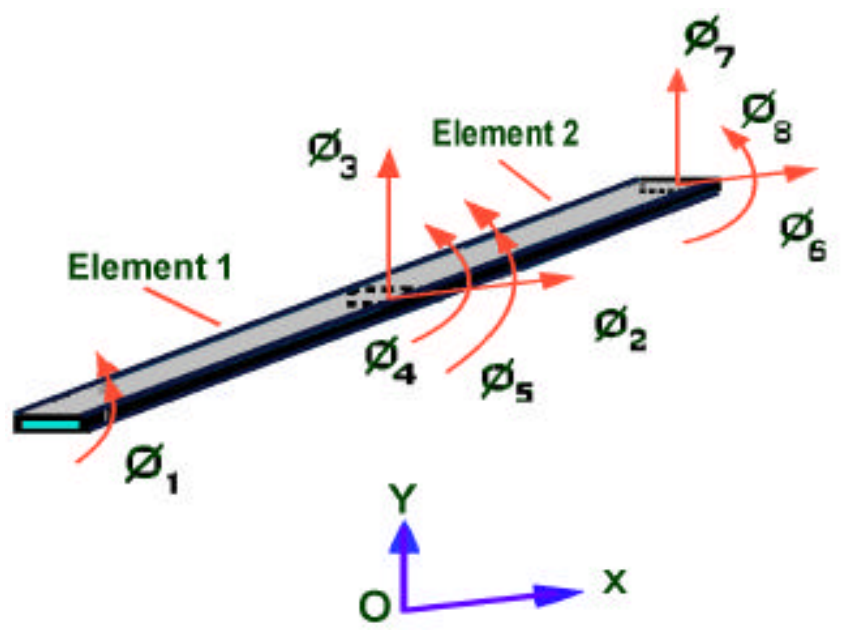

Figure 10. General coordinates of a DLO 
The cross section of the DLO is rectangular. All of the cases are situated in the vertical plane. The gravitational acceleration is $9.80 \mathrm{~m} / \mathrm{s}^{2}$. The physical parameters of the DLO are given in Table 2 .

Table 2. Parameters of the DLO

\begin{tabular}{|c|c|}
\hline length of DLO & $1.0 \mathrm{~m}$ \\
\hline length of element & $0.5 \mathrm{~m}$ \\
\hline width of DLO & $11 \mathrm{~mm}$ \\
\hline height of DLO & $0.5 \mathrm{~mm}$ \\
\hline elastic modules & $1.26 \times 10^{11} \mathrm{~Pa}$ \\
\hline shear modules & $0.70 \times 10^{11} \mathrm{~Pa}$ \\
\hline Density & $8960 \mathrm{Kg} / \mathrm{m}^{3}$ \\
\hline damping coefficient & 0.0005 \\
\hline
\end{tabular}

\subsection{Cases of one-way RAMo}

The following three cases are simulated using a one-way RAMo as the adjustment type. The previous motions of the cases are rotation, translation and combined rotation and translation, respectively.

\subsubsection{Case One}

Suppose that the previous end-effector's motion is rotational. The adjustment-motion begins after the rotation and a certain delay. The process of generating the parameters takes about 30 minutes using a Pentium 500MHz computer. In this case, the GA generated parameters are: delay time $0.047 \mathrm{sec}$, value of acceleration $-59.530 \mathrm{rad} / \mathrm{s}^{2}$, constant angular velocity $-2.401 \mathrm{rad} / \mathrm{s}$, running time at this velocity $0.131 \mathrm{sec}$ and the value of deceleration $50.054 \mathrm{rad} / \mathrm{s}^{2}$. The results are given in the following figures.

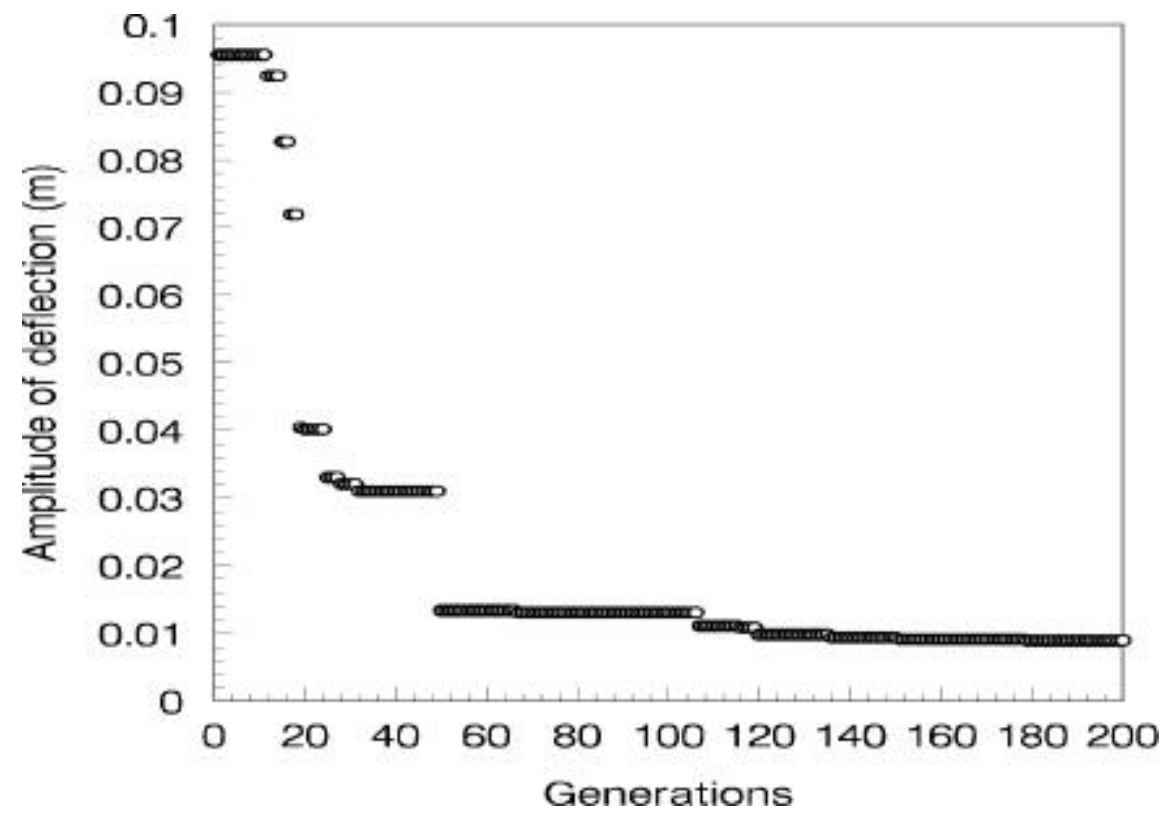

Figure 11. Amplitude of deflection of DLO versus number of generations (Previous motion: Rotation) 
Figure 11 shows the vibrational amplitude versus the number of completed GA generations. The shape, vibration and motion of the DLO with and without adjustment-motion are shown and compared in Figure 12. The vibrational and adjustment-motion portions of the complete motion are both shown again separately in the lower portion of the figure. A detailed comparison of the vibrational amplitudes resulting from these two situations is shown in Figure 13. It was found that the adjustment-motion can effectively reduce the vibrational amplitude.

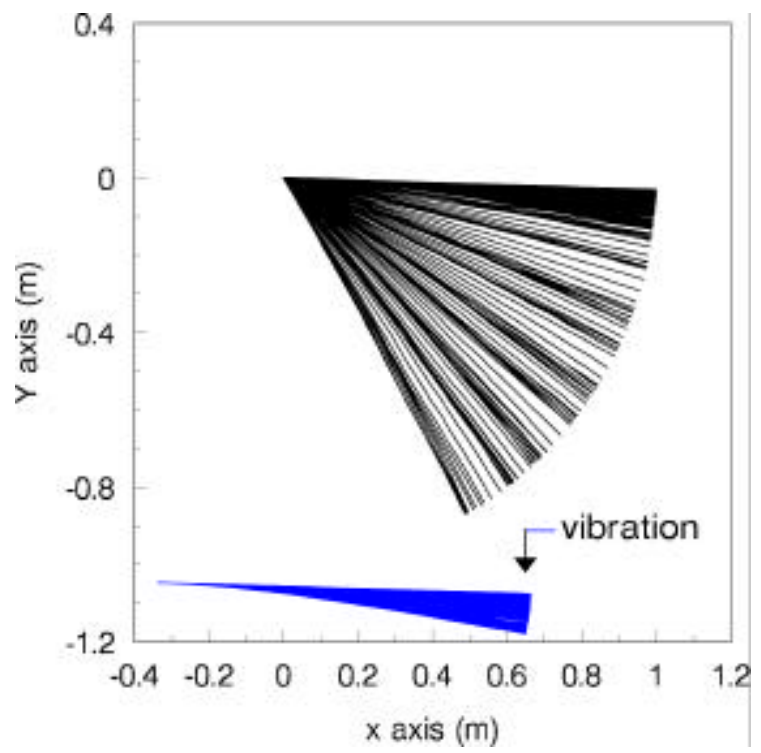

(a). End-effector stops after the rotation

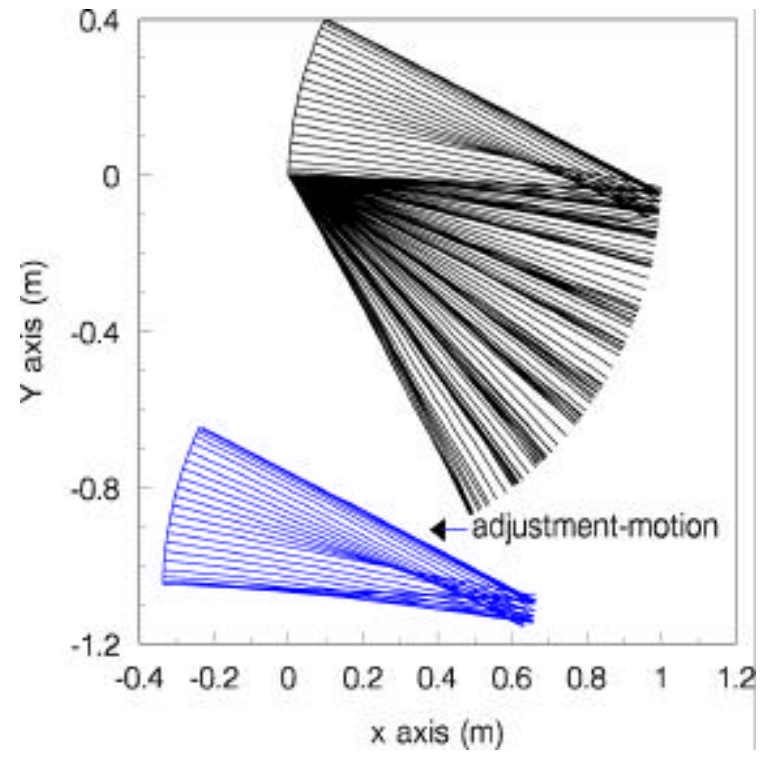

(b). End-effector adjusts after the rotation

Figure 12. The previous motion, vibration and one-way RAMo of the DLO

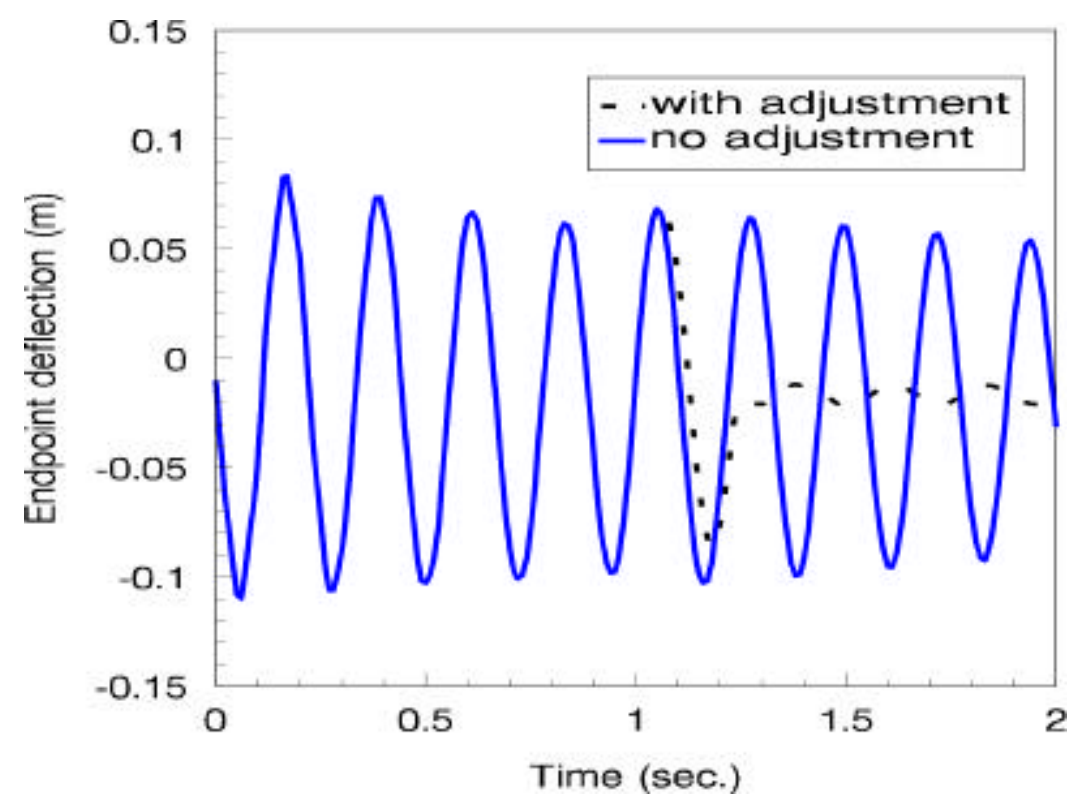

Figure 13. Deflection of the DLO with and without one-way RAMo (Previous motion: Rotation) 


\subsubsection{Case Two}

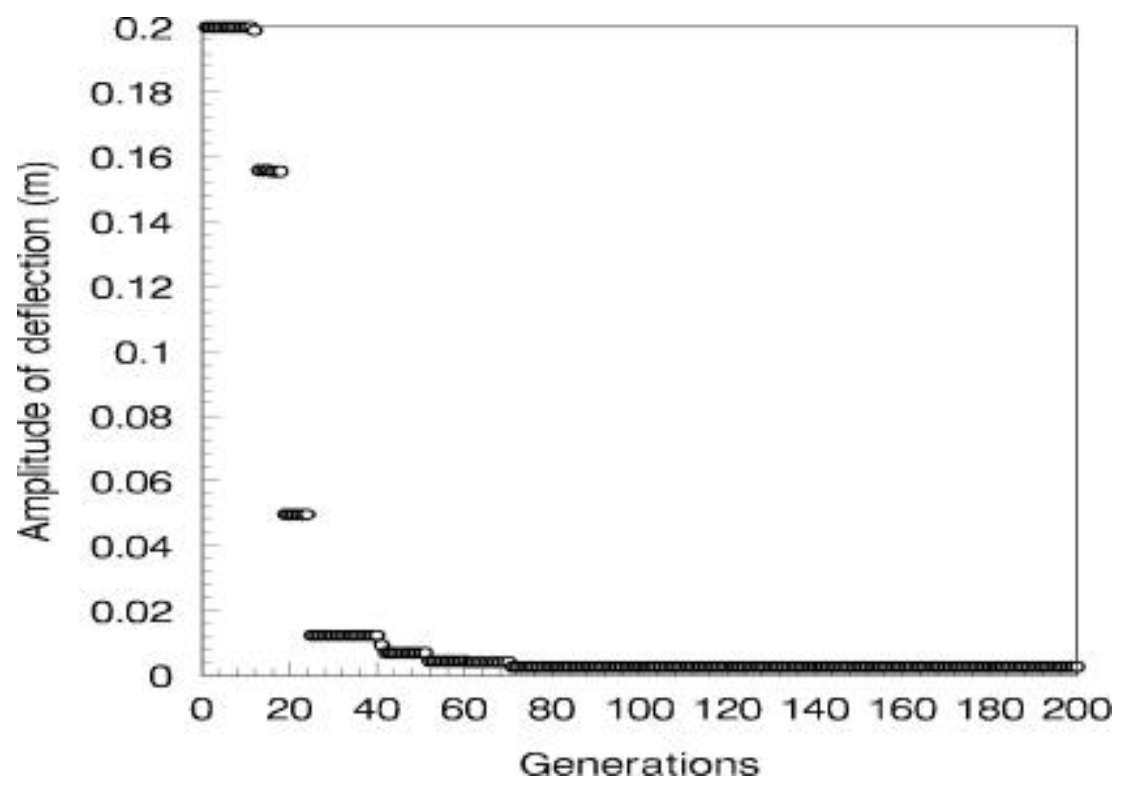

Figure 14. Amplitude of deflection of DLO versus number of generations (Previous motion: Translation)

Suppose the previous end-effector's motion is translation. The adjustment-motion begins after the translation and a certain delay. In this case, the GA generated parameters are: delay time $0.068 \mathrm{sec}$, value of acceleration $-96.481 \mathrm{rad} / \mathrm{s}^{2}$, the constant angular velocity $-3.001 \mathrm{rad} / \mathrm{s}$, the running time at this velocity $0.117 \mathrm{sec}$ and the value of deceleration $56.798 \mathrm{rad} / \mathrm{s}^{2}$. The results are found in the following figures.

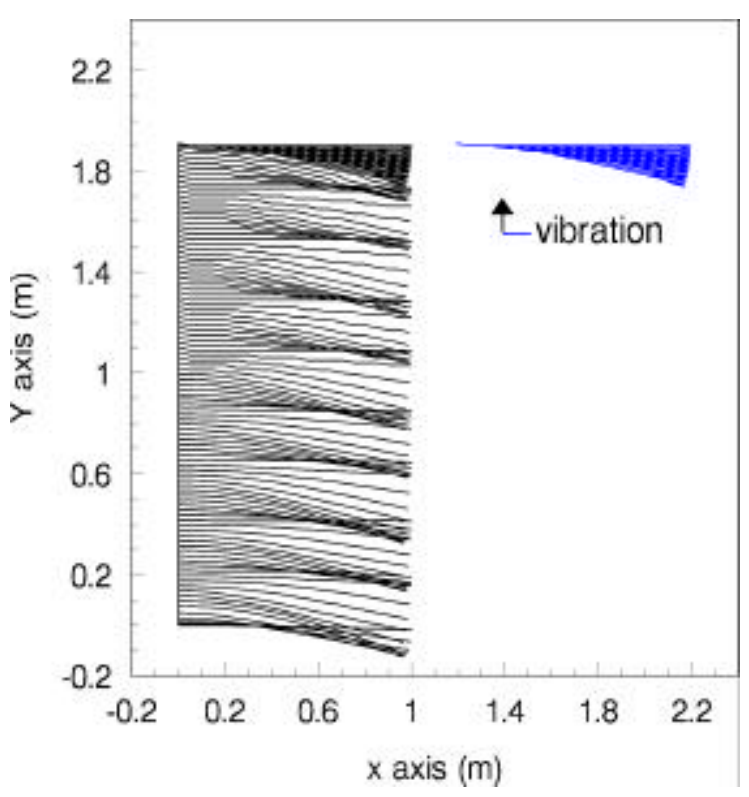

(a).End-effector stops after the translation

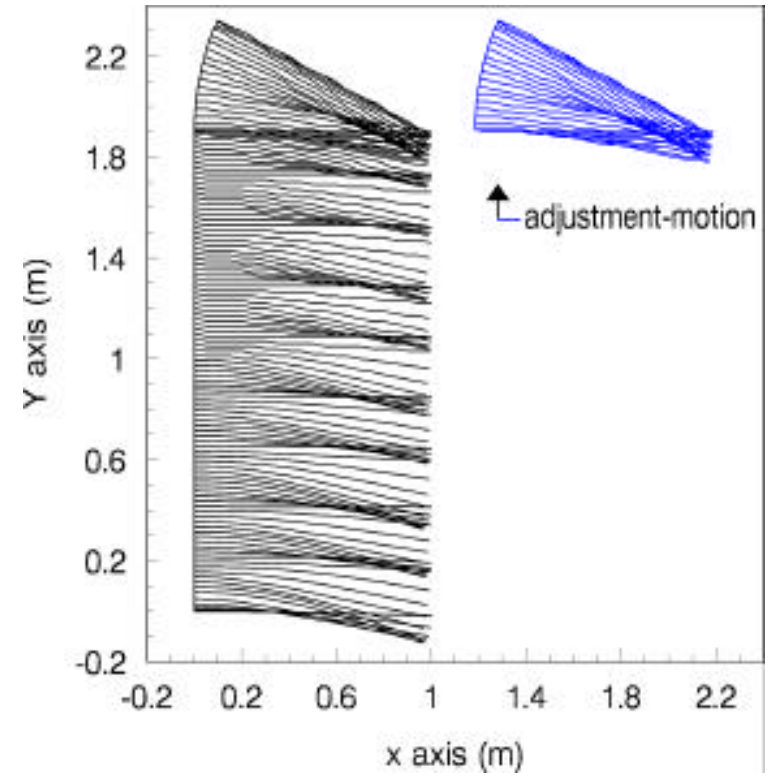

(b). End-effector adjusts after the translation

Figure 15. The previous motion, vibration and one-way RAMo of the DLO 
Figure 14 shows the vibrational amplitude versus number of completed GA generations. The shapes and motions of the DLO with and without an adjustment-motion are shown and compared in Figure 15. A detailed comparison of the vibrational amplitude resulting from these two situations is shown in Figure 16. It was again found that the adjustment-motion can effectively reduce the vibrational amplitude.

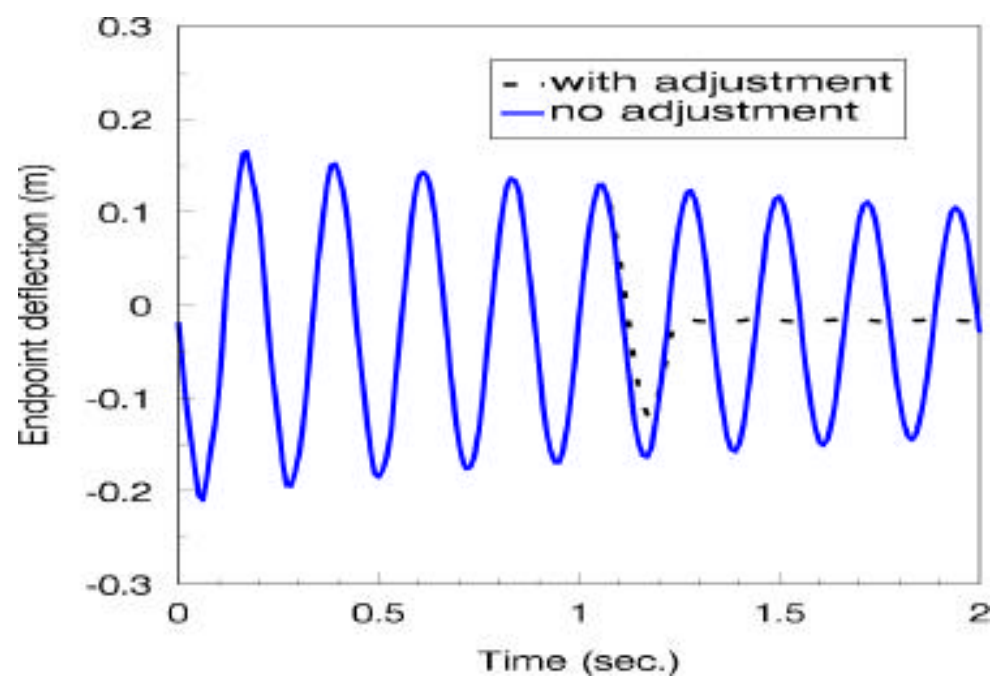

Figure 16. Deflections of the DLO with and without one-way RAMo (Previous motion: Translation)

\subsubsection{Case Three}

To verify the effectiveness of this method based on a more general previous motion, we present here another case study. In this example, the initial motion combines rotation and translation. In case three, the GA generated parameters are: delay time $0.200 \mathrm{sec}$, value of acceleration $-97.263 \mathrm{rad} / \mathrm{s}^{2}$, the constant angular velocity $-4.000 \mathrm{rad} / \mathrm{s}$, the running time at this velocity $0.164 \mathrm{sec}$ and the value of deceleration $57.971 \mathrm{rad} / \mathrm{s}^{2}$.

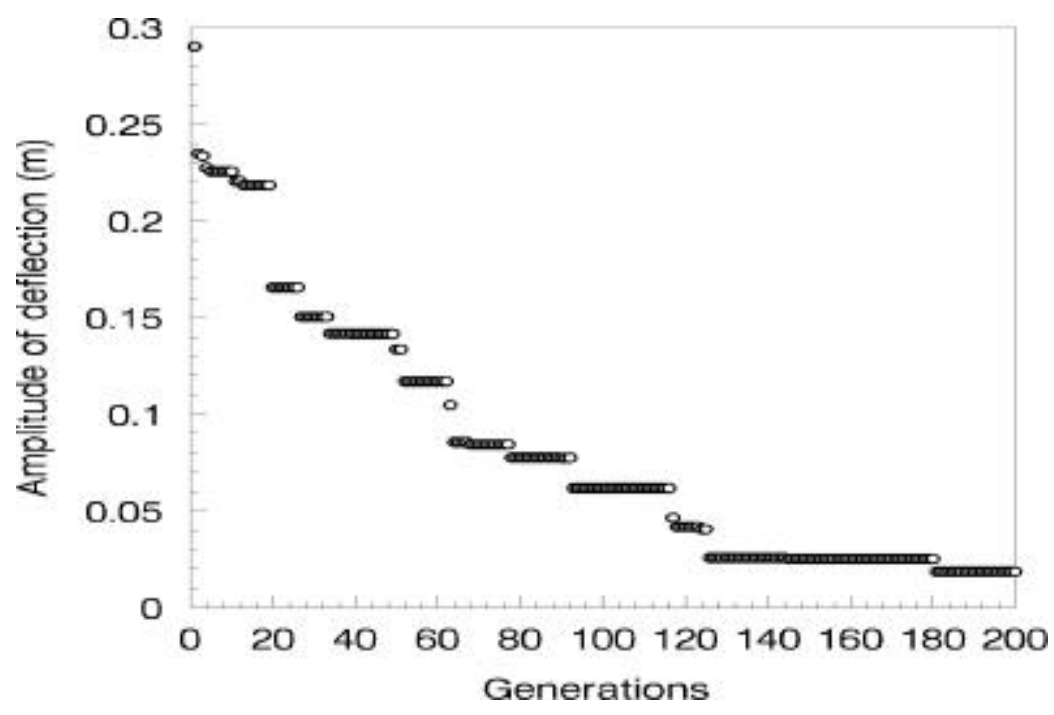

Figure 17. Amplitude of deflection of DLO versus number of generations (Previous motion: Combined rotation and translation) 
The results are shown in Figures 17, 18 and 19. It was found once again that the attached adjustment-motion is effective in reducing vibrations of the DLO. This implies that the adjustment-motion skill can be attached to any complex previous motions.

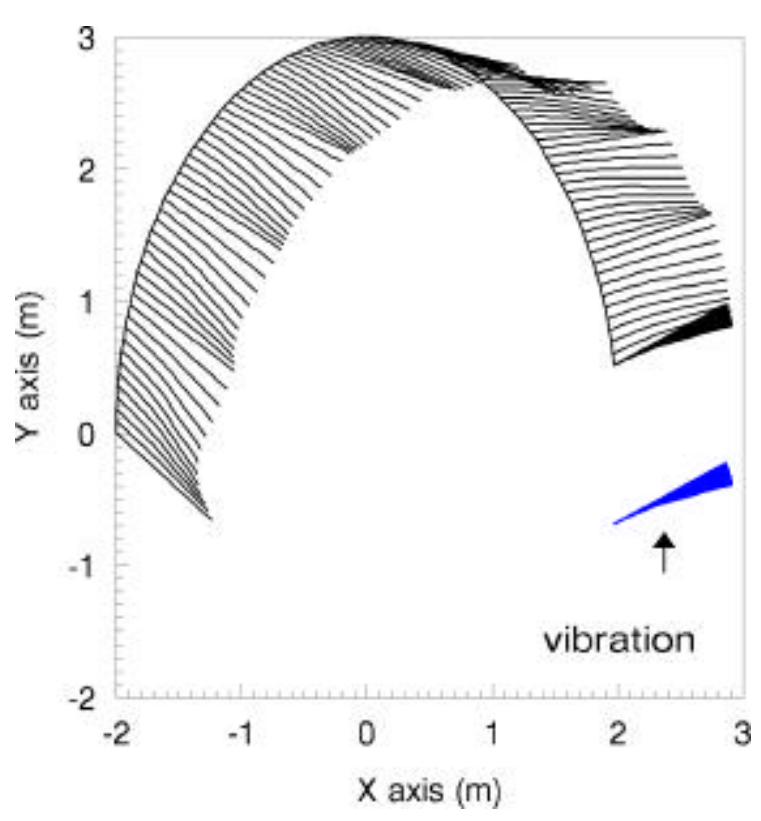

(a). End-effector stops after initial motion

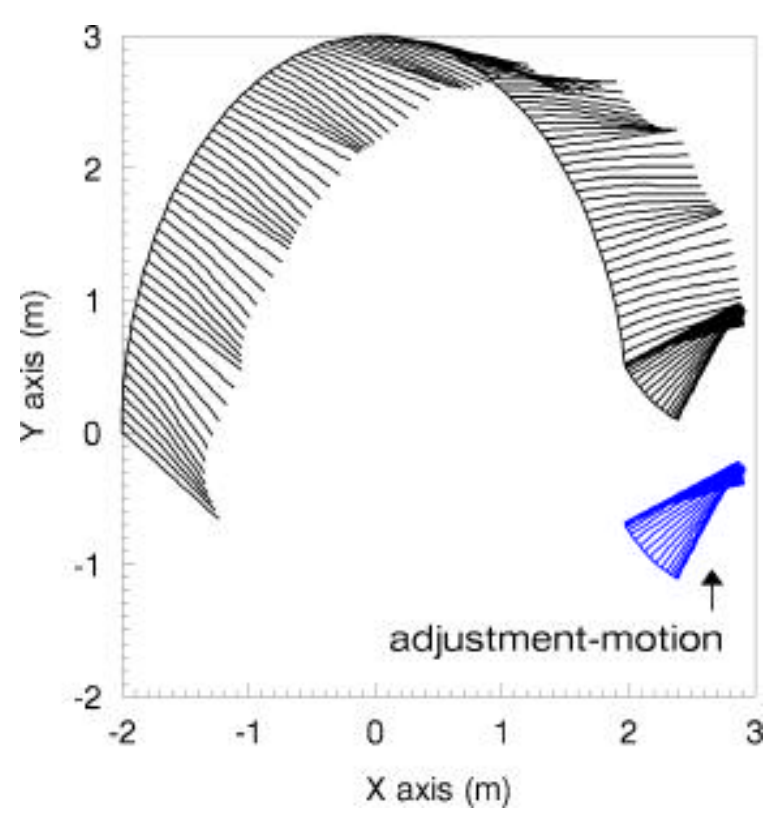

(b). End-effector adjusts after initial motion

Figure 18. The previous motion, vibration and one-way RAMo of the DLO

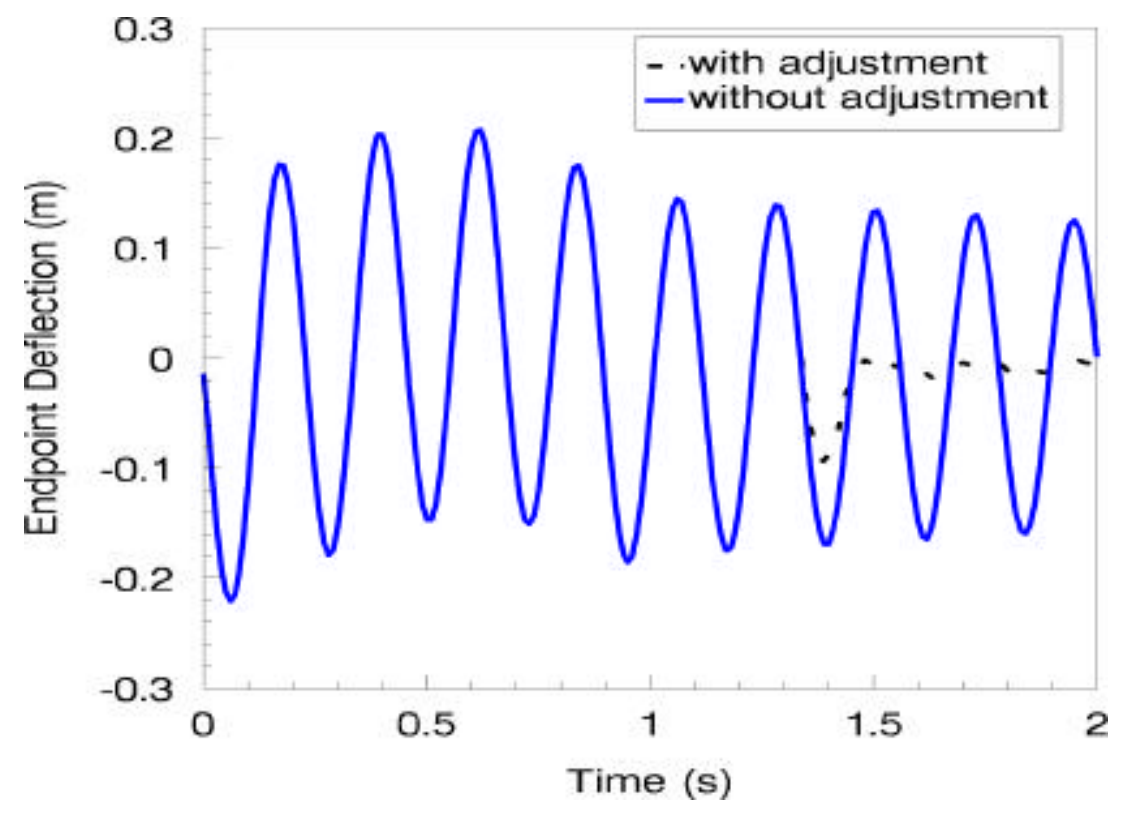

Figure 19. Deflections of DLO with and without adjustment-motion (Previous motion: Combined rotation and translation) 
The above cases show the effectiveness of one-way RAMos. How about the other adjustmentmotion styles? In the following cases, different styles are used to reduce vibration caused by the same previous motion. A combined rotation and translation is used as previous motion in the following cases.

\subsubsection{One-way TAMo}

In this case, TAMo is chosen as the adjustment style. The GA generated parameters are: delay time $0.125 \mathrm{sec}$, value of acceleration $-39.198 \mathrm{~m} / \mathrm{s}^{2}$, the constant velocity $-2.023 \mathrm{~m} / \mathrm{s}$, the running time at this velocity $0.055 \mathrm{sec}$ and the value of deceleration $52.551 \mathrm{rad} / \mathrm{s}^{2}$. The results are shown in Figure 20 to Figure 22. It was found that the method using a TAMo is quite effective.

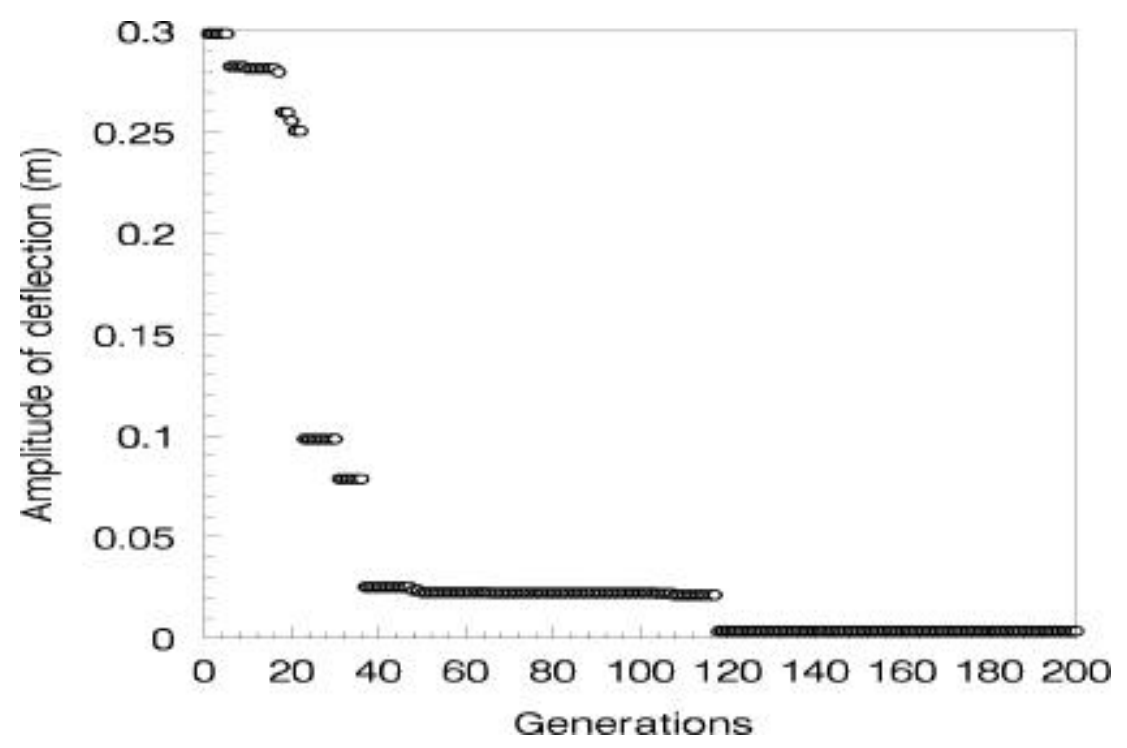

Figure 20. Amplitude of deflection of DLO versus number of generations by using one-way TAMo

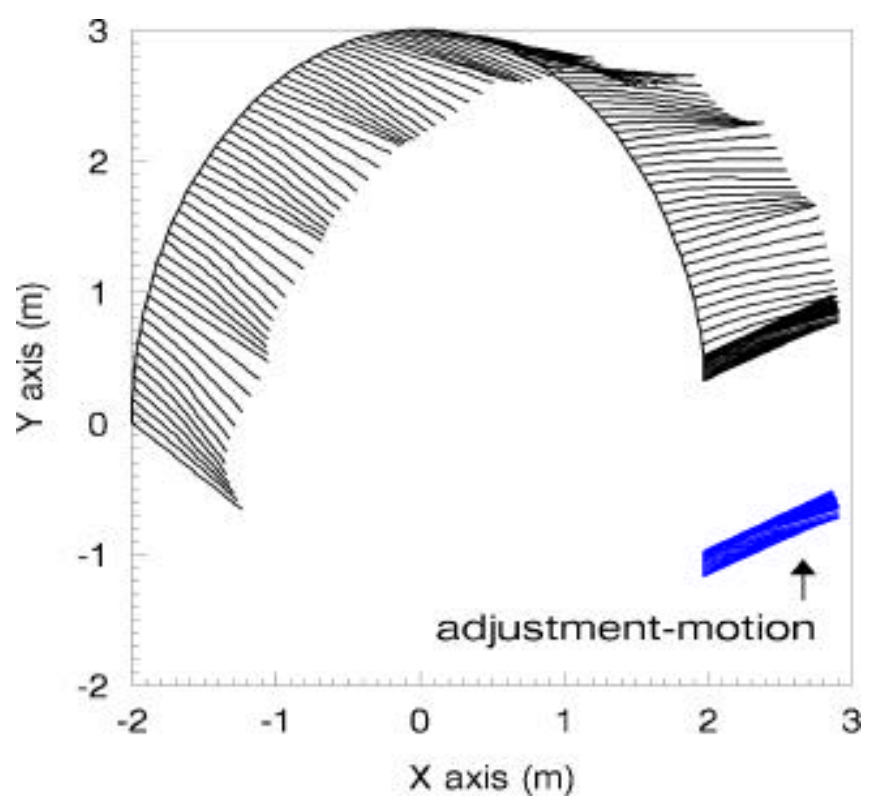

Figure 21. The previous motion and one-way TAMo of the DLO 


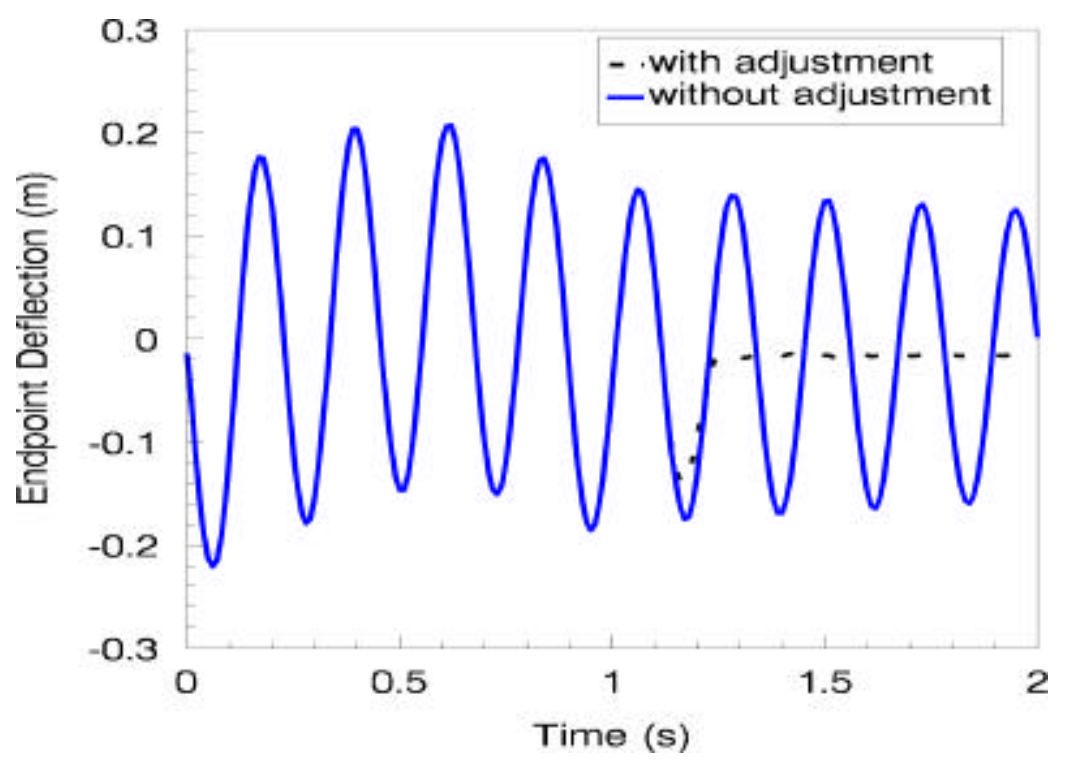

Figure 22. Deflection of the DLO with and without one-way TAMo

\subsubsection{Two-way RAMo}

In this two-way case study, RAMo is chosen as the adjustment style. The GA generated parameters are: delay time $0.342 \mathrm{sec}$, value of acceleration $61.486 \mathrm{rad} / \mathrm{s}^{2}$, the constant angular velocity $3.867 \mathrm{rad} / \mathrm{s}$, the running time at this velocity $0.012 \mathrm{sec}$ and the value of deceleration $-91.829 \mathrm{rad} / \mathrm{s}^{2}$. The results are given in Figure 23 to Figure 25 respectively. It was found that the two-way RAMo is also effective.

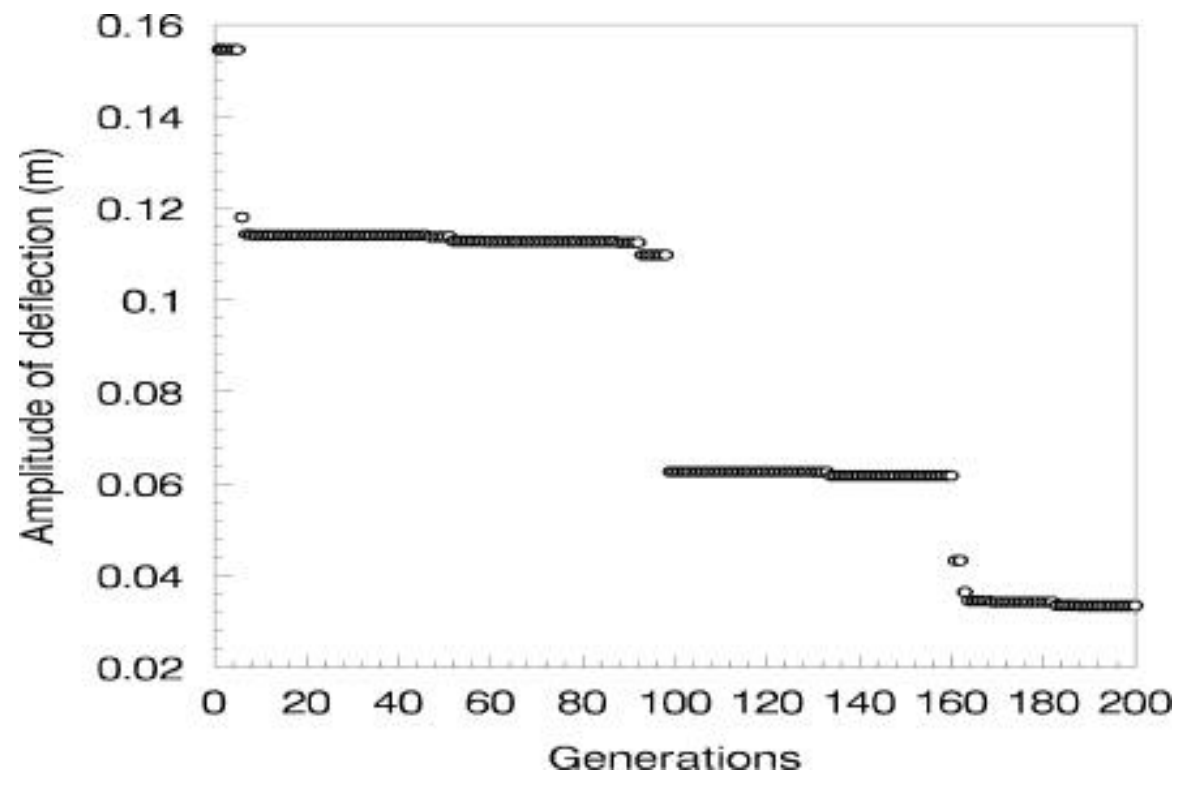

Figure 23. Amplitude of deflection DLO versus number of generations by using Two-way RAMo 


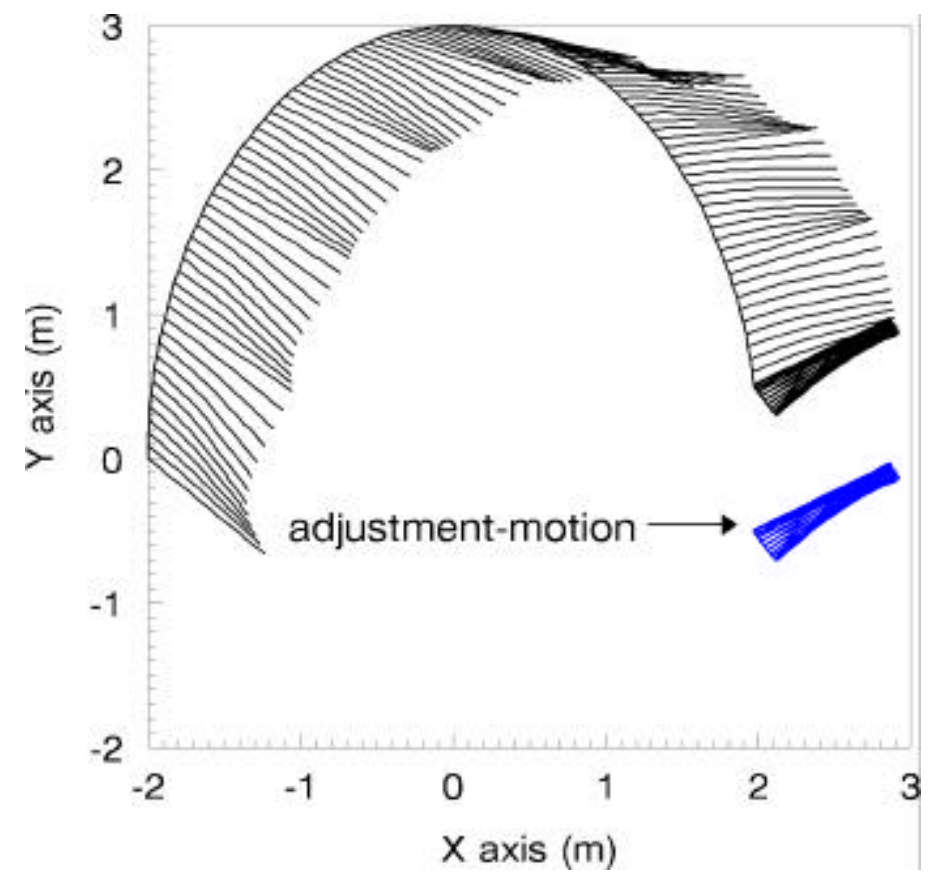

Figure 24. The previous motion and two-way RAMo of the DLO

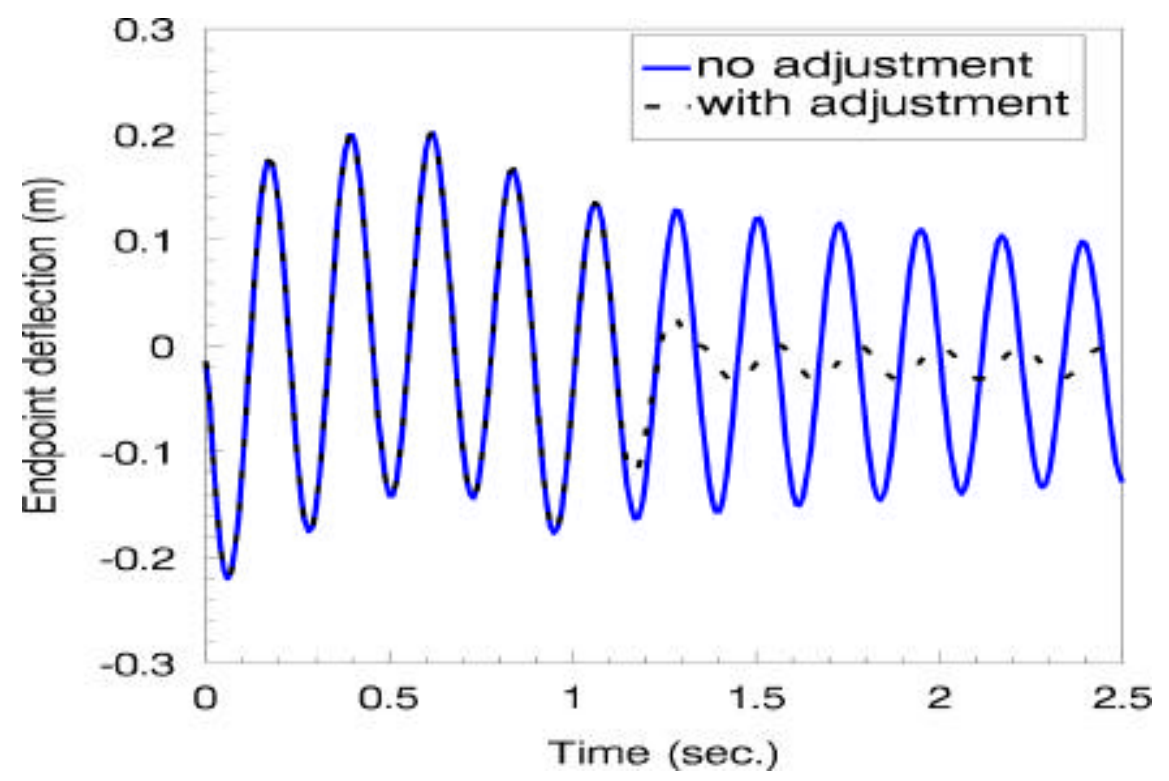

Figure 25. Deflection of the DLO with and without two-way RAMo

\subsubsection{Two-way TAMo}

In this two-way case study, TAMo is chosen as the adjustment style. The GA generated parameters are: delay time $0.248 \mathrm{sec}$, value of acceleration $74.585 \mathrm{~m} / \mathrm{s}^{2}$, the constant velocity $1.114 \mathrm{~m} / \mathrm{s}$, the running time at this velocity $0.095 \mathrm{sec}$ and the value of deceleration 
$-41.409 \mathrm{~m} / \mathrm{s}^{2}$. The results are given in the following figures. It was found that the two-way TAMo is quite effective in reducing vibration resulted from previous motion.

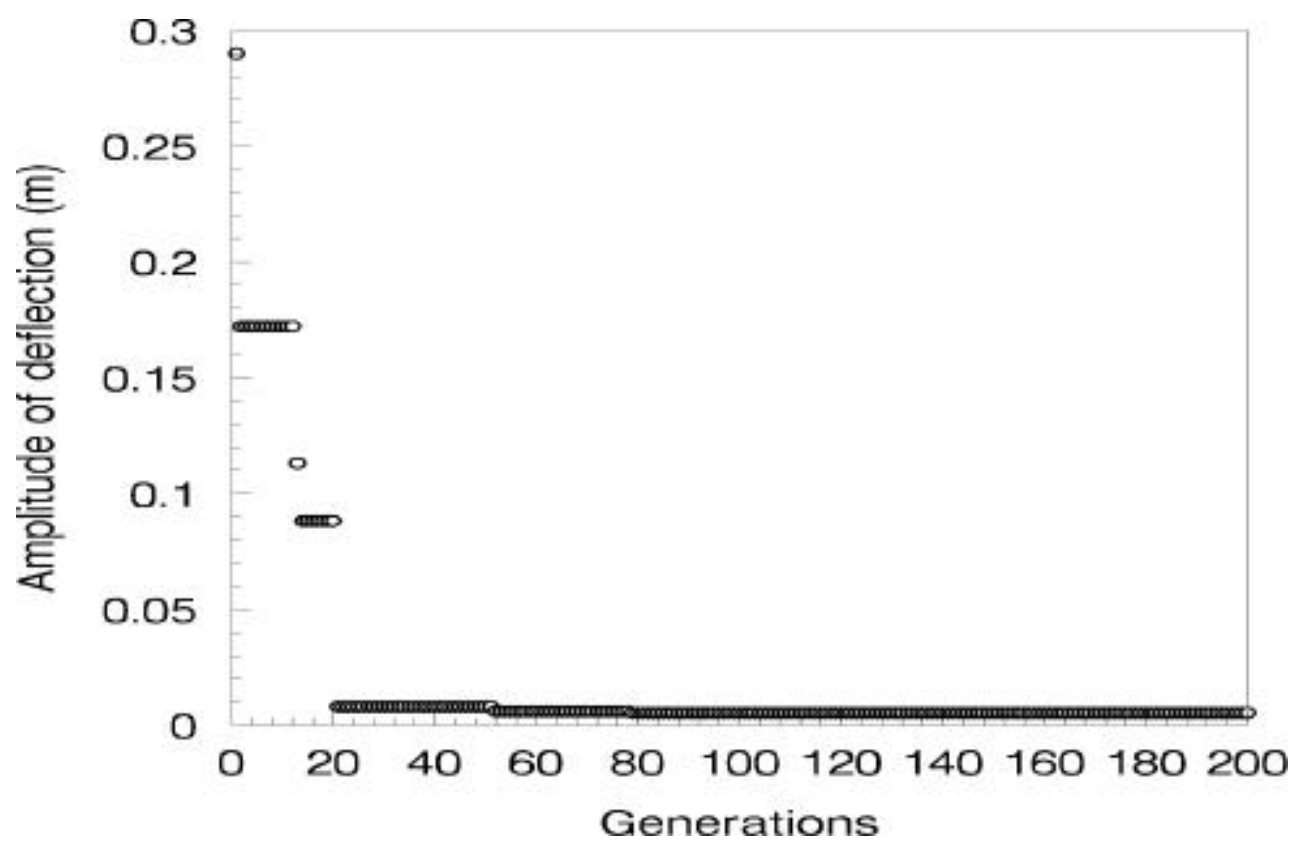

Figure 26. Amplitude of deflection of DLO versus number of generations by using two-way TAMo

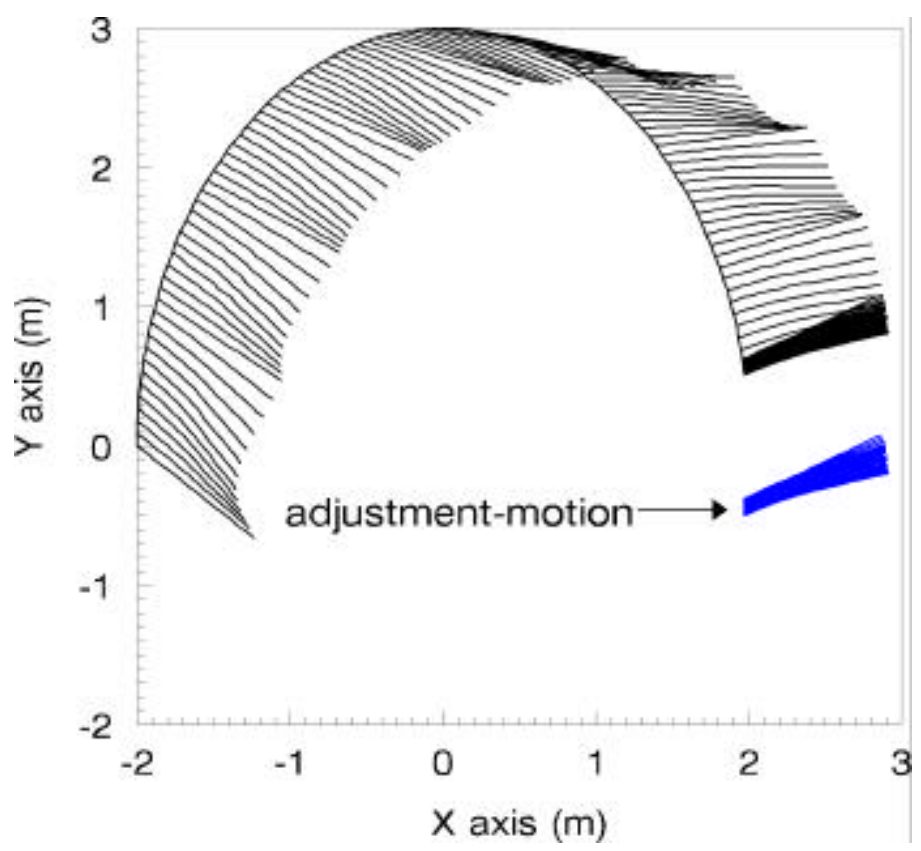

Figure 27. The previous motion and two-way TAMo of the DLO 


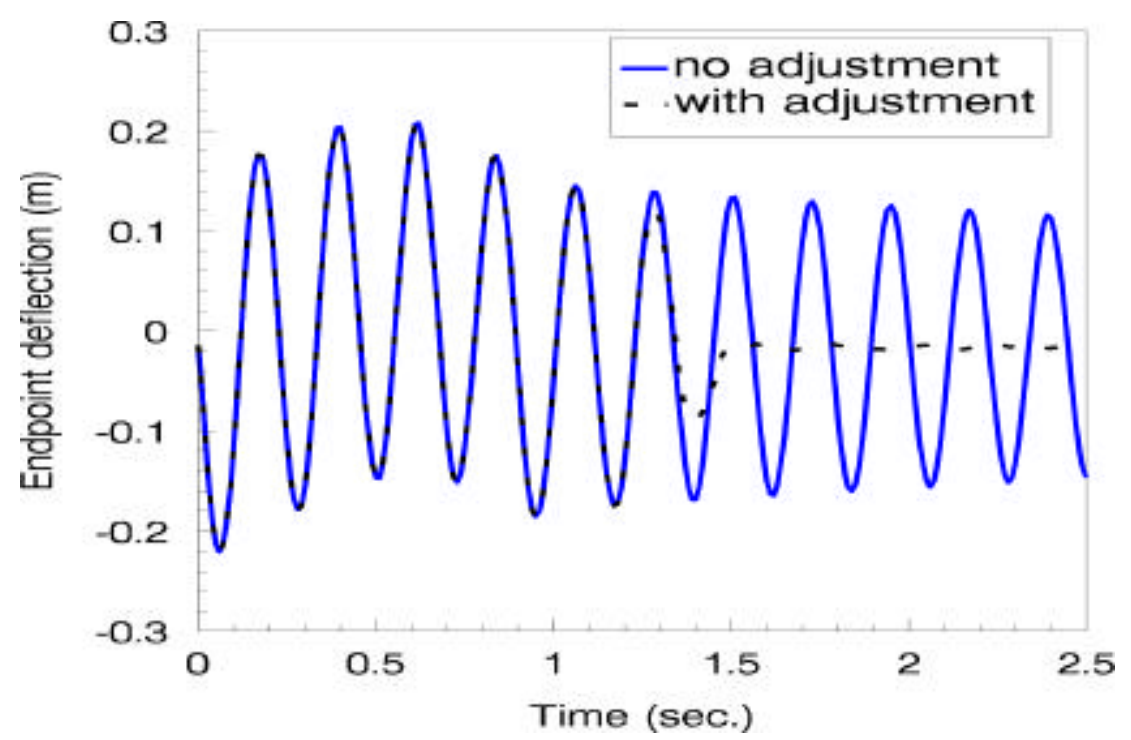

Figure 28. Deflection of the DLO with and without two-way TAMo

The above cases proved that for the same previous motion, each style can decrease vibration amplitude efficiently with slight differences in the results. Which one can be used in practice is probably decided by the environment in which the method is to be applied.

\section{Experiment}

To verify the method presented and simulated above, an experiment was conducted using a Stäubli RX130 industrial robot (Figure 29). A standard 500mm stainless ruler is used as the DLO in the experiment. Considering that one end of the ruler is grasped by the jaws, the actual length of the ruler is $490 \mathrm{~mm}$. The cross section of the ruler is $0.5 \mathrm{~mm} \times 18 \mathrm{~mm}$. A density of $7880 \mathrm{~kg} / \mathrm{m}^{3}$ and an elastic modulus of $368 \times 10^{6} \mathrm{~N} / \mathrm{m}^{2}$ are used in the simulation. The previous motion is assumed to be a translation of $600 \mathrm{~mm}$ within one second and the adjustment-motion style is taken to be one-way TAMo. The experiment is conducted in the horizontal plane.

First, the adjustment-motion is generated by simulation. After 60 generations, the simulation results are obtained. The GA generated parameters are: $0.357 \mathrm{sec}$ delay time, $8.867 \mathrm{~m} / \mathrm{s}^{2}$ acceleration, $0.724 \mathrm{~m} / \mathrm{s}$ velocity, $0.046 \mathrm{sec}$ running time at this velocity and $-8.517 \mathrm{~m} / \mathrm{s}^{2}$ deceleration. Then, these parameters are downloaded to the RX130 control unit with real-time V+ language. The maximum vibrational amplitude obtained by simulation, experiment and handimprovement are compared in Figure 30.

It can be found that all of the vibrational amplitudes are decreased sharply by the adjustmentmotions. However, with the same adjustment, the amplitude of the experiment results is almost two times larger than that of the simulation results. This probably happens for two reasons: model errors and robot limited resolution. Model errors include the error resulting from the method and the error caused by parameter measuring of the DLO. Figure 30 also indicates this aspect, showing a comparison of vibrational amplitudes without adjustment. As to the second source of error, every robot has its limited capabilities when operating under definite demands. Therefore, hand-improved results are also shown in the Figure 30. The hand-improved results are obtained by extending the delay time to $0.4 \mathrm{~s}$, the constant velocity to $0.750 \mathrm{~m} / \mathrm{s}$ and both acceleration and deceleration to $9.000 \mathrm{~m} / \mathrm{s}^{2}$. 
Since the slight changes around the GA generated parameters did not result in sharp changes of the vibrational amplitude of the DLO, the hand-improved results also show the robustness of the attachable adjustment-motion.

Based on the obtained results and discussion of the experiment, the presented method has been proved to be applicable in practice.

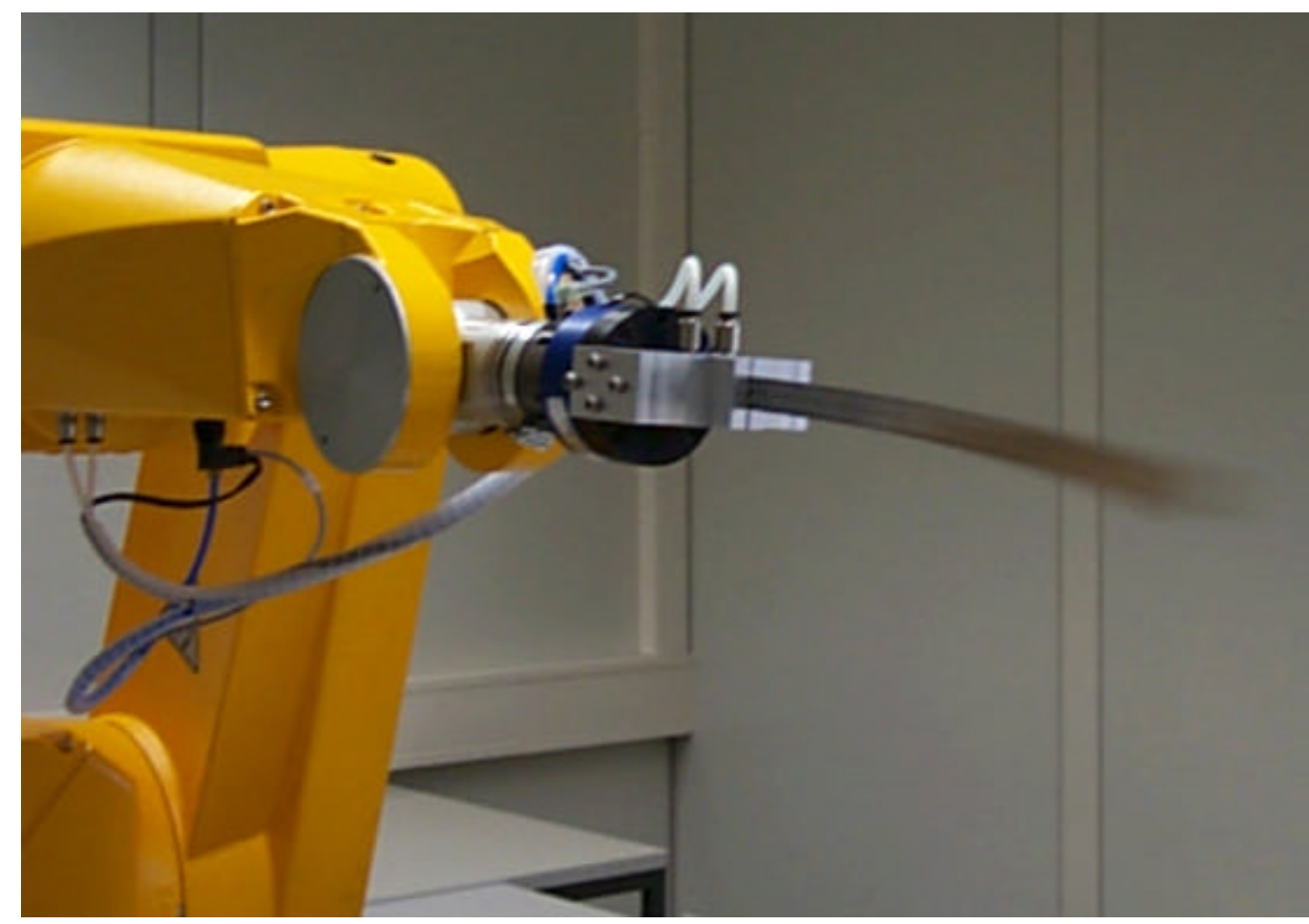

Figure 29. One-way TAMo of the DLO by the end-effector of a $R \times 130$ robot

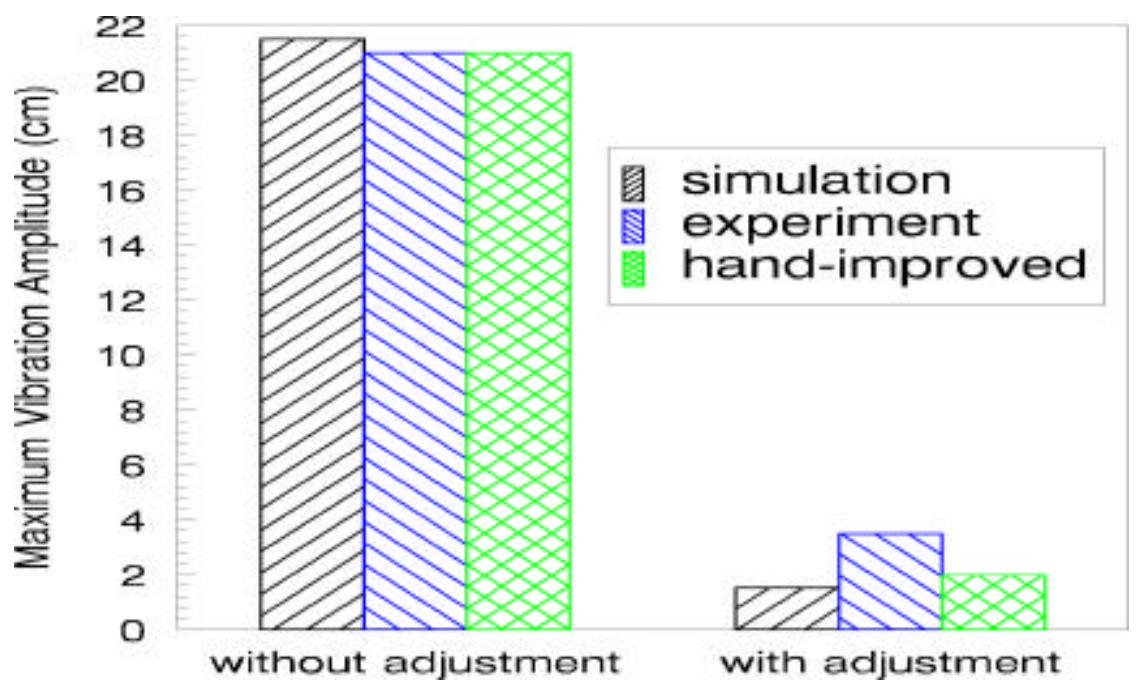

Figure 30. Comparison of results from simulation and experiment 


\section{Conclusion}

In this paper, we have addressed the problem of manipulating deformable linear objects in a way suitable to avoid acute vibration. Different kinds of adjustment-motions have been presented, which can be attached to the end of any arbitrary end effector's trajectory in order to eliminate unwanted vibration of the object. For describing the dynamics of deformable linear objects, finite element method was used to derive the dynamic differential equations. Genetic algorithm was applied to find the optimal adjustment-motion for each simulation example. The case studies showed that adjustment-motion is suitable for eliminating vibration arising during handling of deformable linear objects. An experiment was also conducted and verified the presented method.

Detailed information about previous motions is necessary since sensors are not used in the presented open-loop method. In the future, a new sensor-based method which can utilize sensors to determine the adjustment-motion will be presented. The sensor-based method can be attached and computed on-line instead of off-line. The information about the previous motion will not be necessary if the sensor-based method is employed.

\section{Acknowledgment}

The support of the Alexander von Humboldt Foundation is greatly appreciated by the first author. We would like to thank Mr. Dirk EBERT and Mr. Thorsten SCHMIDT for their kind help in preparing pictures.

\section{References}

1. A. Villarreal, and H. Asada, A geometric representation of distributed compliance for the assembly of flexible parts, Proc. IEEE Int. Conf. on Robotics and Automation, 1991, pp.2708-2715.

2. H. Nakagaki, K. Kitagaki, T. Ogasawara, and H. Tsukune, Study of insertion task of a flexible beam into a hole by using visual tracking observed by stereo vision, Proc. of IEEE Int. Conf. on Robotics and Automation, Minneapolis, USA, April, 1996, vol.4, pp.3209-3214.

3. H. Nakagaki, K. Kitagaki, T. Ogasawara, and H. Tsukune, Study of deformation and insertion task of a flexible wire, Proc. of IEEE Int. Conf. On Robotics and Automation, (ICRA'97) , , Albuquerque, USA, April, 1997, vol.3, pp.2397-2402.

4. H. Wakamatsu, T. Matsumura, E. Arai, and S. Hirai, Dynamic analysis of rod like object deformation towards their dynamic manipulation, Proc. of IEEE/IROS Int. Conf. on Intelligent Robots and Systems, 1997, pp.196-201.

5. H. Wakamatsu, S. Hirai, and K. Iwata, Static analysis of deformable object grasping based on bounded force closure, Proc. IEEE Int. Conf. on Robotics and Automation, Minneapolis, April, 1996, vol.4, pp.3324-3329.

6. K. Higashijima, H. Onda, and T. Ogasawara, Planning for wire obstacles avoidance using ultrasonic sensors, In $8^{\text {th }}$ Int. Conf. on Advance Robotics (ICRA'97), California, July 7-9, 1997, pp.577-582.

7. P.W. Simth, N. Nandhakumar, and A.K. Tamadorai, Vision based manipulation of non rigid objects, Proc. IEEE Robotics and Automation, Minneapolis, Minnesota, USA, April, 1996, vol.4, pp.3191-3196.

8. S. Hirai, H. Wakamatsu, and K. Iwata, Modeling of deformable thin parts for their manipulation, Proc. IEEE Int. Conf. on Robotics and Automation, San Diego, USA, May, 1994, vol.4, pp.2955-2960.

9. S. Hirai, H. Noguchi, and K. Iwata, Transplantation of human skillful motion to manipulators in insertion of deformable tubes, Proc. IEEE Int. Conf. on Robotics and Automation, Nagoya, Japan, 21-27 May, 1995, vol.2, pp.1900-1905. 
10. S. Hirai, and H. Wakamatsu, Modeling of deformable strings with bend, twist, and extension in 3D space, Proc. $2^{\text {nd }}$ ECPD int. Conf. on Advanced Robotics, Intelligent Automation and Active Systems, Vienna, Sept. 1996, pp.529-534.

11. S. Hirai, H. Noguchi, and K. Iwata, Human-demonstration based approach to the recognition of process state transitions in insertion of deformable tubes, Proc. IEEE Int. Conf. Robotics and Automation, Minneapolis, USA, April, 1996, vol.3, pp.2006-2011.

12. T. Yukawa, M. Uchiyama, D.N. Nenchev, and H. Inooka, Stability of control system in handling of a flexible object by rigid arm robots, Proc. IEEE Int. Conf. on Robotics and Automation, Minneapolis, USA, April, 1996, pp.2332-2338.

13. Y.F. Zheng, R. Pei, and C. Chen, Strategies for automatic assembly of deformable objects, Proc. IEEE Int. Conf. On Robotics and Automation, 1991, pp.2598-2603.

14. T. Hasegawa, T. Suehiro, and T. Takase, A model-based manipulation system with skillbased execution, IEEE Trans. On Robotics and Automation, 8(5)(1992) 535-544.

15. H. Onda, H. Hirukawa, and K. Takase, Assembly motion teaching system using position/force simulator -extracting a sequence of contact state transition, Proc. of the IEEE/RSJ Int. Conf. on Intelligent Robots and Systems, 1995, vol.1, pp.9-16.

16. D. Henrich, T. Ogasawara, and H. Wörn, Manipulating deformable linear objects - contact states and point contacts - , IEEE Int. Symposium on Assembly and Task Planning (ISATP'99), Porto, Portugal, July 21-24, 1999.

17. F. Abegg, A. Remde, and D. Henrich, "Force and vision based detection of contact state transitions," Robot Manipulation of Deformable Objects, D. Henrich and H. Wörn (Editor), Springer-Verlag, London, 2000.

18. A. Remde, D. Henrich, and H. Wörn, Picking-up deformable linear objects with industrial robots, Int. Symposium on Robotics (30 ${ }^{\text {th }}$ ISR), Tokyo, Japan, Oct.27-29, 1999.

19. M.Z. Chen, and Y.F. Zheng, Vibration-Free handling of deformable beams by robot endeffectors, J. of Robotics Systems, , 12(5) (1995) 331-347.

20. J.F. Jones and B.J. Petterson, Oscillation damped movement of suspended objects, Proc. IEEE Int. Conf. on Robotics and Automation, 1988, pp.956-962.

21. G.P. Starr, Swing-free transport of suspended objects with a path-controlled robot manipulator, J. Dyn. System Control Trans. ASME, 1985, 97-100.

22. W.L. Cleghorn, R.G. Fenton, and B. Tabarrok, Finite element analysis of high-speed flexible mechanisms, Mechanism and Machine Theory, 16(4) (1981) 407-424.

23. S.G. Yue, Y.Q. Yu, and S.X. Bai, Flexible rotor beam element for manipulators with joint and link flexibility, Mechanism and Machine Theory, 32(2)(1997) 209-219.

24. D.B. Fogel, An introduction to simulated evolutionary optimization, IEEE Trans. on Neural Networks, 5(1)(1994) 3-14.

25. D.E. Goldenberg, Genetic Algorithms in search, optimization and machine learning, AddisonWesley, Reading, Mass., 1989

26. N. Kubota, T. Arakawa, T. Fukuda, and K. Shimojima, Trajectory generation for redundant manipulator using virus evolutionary genetic algorithm, Proc. IEEE Int. Conf. on Robotics and Automation, 1997, pp.205-210.

27. S.D. Sun, A.S. Morris, and A.M.S. Zalzala, Trajectory planning of multiple coordinating robots using genetic algorithms, Robotica, 14(1996) 227-234. 


\section{Appendix}

$$
\vec{D}_{x}=\left[\begin{array}{c}
1-\frac{1}{L} x \\
0 \\
0 \\
0 \\
\frac{1}{L} x \\
0 \\
0 \\
0
\end{array}\right]_{8 \times 1} \vec{D}_{y}=\left[\begin{array}{c}
0 \\
1-\frac{10}{L^{3}} x^{3}+\frac{15}{L^{4}} x^{4}-\frac{6}{L^{5}} x^{5} \\
x-\frac{6}{L^{2}} x^{3}+\frac{8}{L^{3}} x^{4}-\frac{3}{L^{4}} x^{5} \\
\frac{1}{2} x^{2}-\frac{3}{2 L} x^{3}+\frac{3}{2 L^{2}} x^{4}-\frac{1}{2 L^{3}} x^{5} \\
\frac{10}{L^{3}} x^{3}-\frac{15}{L^{4}} x^{4}+\frac{6}{L^{5}} x^{5} \\
-\frac{4}{L^{3}} x^{3}+\frac{7}{L^{4}} x^{4}-\frac{3}{L^{5}} x^{5} \\
\frac{1}{2 L} x^{3}-\frac{1}{L^{2}} x^{4}+\frac{1}{2 L^{3}} x^{5}
\end{array}\right]_{8 \times 1}
$$

